\title{
Civilisations
}

Revue internationale d'anthropologie et de sciences

humaines

60-1|2011

Mobilisations et dynamiques identitaires en Amérique andine

\section{Appropriation des ressources « naturelles » et criminalisation des communautés paysannes}

Le cas du Rufiji, Tanzanie

Jean-Luc Paul, Stéphanie Duvail et Olivier Hamerlynck

\section{(2) OpenEdition}

\section{Journals}

Édition électronique

URL : http://journals.openedition.org/civilisations/2806

DOI : $10.4000 /$ civilisations. 2806

ISSN : 2032-0442

Éditeur

Institut de sociologie de l'Université Libre de Bruxelles

Édition imprimée

Date de publication : 31 décembre 2011

Pagination : 143-175

ISBN : 2-87263-035-X

ISSN : 0009-8140

\section{Référence électronique}

Jean-Luc Paul, Stéphanie Duvail et Olivier Hamerlynck, « Appropriation des ressources « naturelles » et criminalisation des communautés paysannes », Civilisations [En ligne], 60-1 | 2011, mis en ligne le 12 février 2015, consulté le 01 mai 2019. URL : http://journals.openedition.org/civilisations/2806 ; DOI : 10.4000 /civilisations. 2806 


\title{
Appropriation des ressources «naturelles » et criminalisation des communautés paysannes \\ Le cas du Rufiji, Tanzanie ${ }^{1}$
}

\author{
Jean-Luc PAUL, Stéphanie DUVAIL et Olivier HAMERLYNCK
}

\begin{abstract}
Résumé : La plaine inondable du Rufiji (Tanzanie) est occupée par des populations dont les activités économiques principales sont l'agriculture vivrière et la pêche commerciale. Les revenus de la pêche légale assurent avec peine la sécurité alimentaire des foyers mais ne permettent pas de faire face à des dépenses " exceptionnelles ». Ainsi, pour financer les études secondaires de leurs enfants, les villageois n'ont guère d'autre choix que de quitter les zones de pêches légales et de pénétrer, au risque de leur vie, la Réserve de Chasse du Selous (50 $\left.000 \mathrm{~km}^{2}\right)$ où se trouvent de nombreux lacs poissonneux. La Réserve, située à proximité immédiate des villages, s'est constituée progressivement au cours du XXe siècle par une série de décisions autoritaires et coercitives. Les communautés locales ne bénéficient d'aucune manière des revenus substantiels issus de son exploitation tandis qu'elles en supportent l'essentiel des coûts induits. Les nouvelles politiques de conservation " participatives » (community based conservation) mises en place depuis une dizaine d'années restent à l'état de déclarations d'intention.
\end{abstract}

Mots-clés : Tanzanie, Rufiji, conservation, ressources naturelles, communautés locales, anthropologie économique.

Abstract: In Tanzania, the Rufiji floodplain is populated by people earning their living from agriculture and fishing. Revenues from legal fishing hardly secure food security of households, and don't really allow for other investments or expenses. In order for instance to earn enough money to pay for secondary school fees, many villagers actually need to leave the legal fishing zones and to penetrate in the Selous Reserve, where several lakes abounding in fish are situated. The Reserve has been progressively constituted throughout the twentieth century, but local villagers do not beneficiate from the revenues it generates. New "participatory" approaches and conservation policies are only declarations of interest without concrete effects.

Keywords: Tanzania, Rufiji, conservation policies, natural resources, local communities, economic anthropology.

1. Remerciements aux évaluateurs de la revue dont les suggestions et les orientations bibliographiques complémentaires ont permis d'enrichir et de mieux organiser notre propos. 


\section{Introduction}

Ce papier raconte avant tout une histoire, celle d'êtres humains qui vivent dans la situation tragique de devoir risquer régulièrement leur vie afin de payer la scolarisation de leurs enfants... De ce point de vue, il est une simple contribution ethnographique à la connaissance de sociétés rurales africaines contemporaines pour lesquelles la modernisation, c'est-à-dire l'intégration à la société globale du marché mondial, est une longue et lente involution. Les informations qu'il propose sont issues du long séjour ${ }^{2}$ d'un ethnologue au sein d'un village tanzanien situé le long du fleuve Rufiji à proximité immédiate d'une immense aire protégée, la Réserve du Selous. Aux témoignages recueillis grâce aux amitiés nouées et à la confiance gagnée, s'ajoutent, outre les observations directes, des données quantitatives basées sur les suivis quotidiens et pluriannuels de deux échantillons indépendants ( 9 foyers et 7 pêcheurs) et de nombreuses enquêtes. Ce type d'informations quantitatives devient de plus en plus rare, car les conditions institutionnelles de la recherche rendent désormais difficile, au-delà d'une thèse de doctorat, un travail de terrain aussi long et fouillé. La nécessité de publier rapidement vient sans doute encore renforcer cette difficulté.

Bien que le partage de ce travail ethnographique avec la communauté scientifique nous paraisse souhaitable en soi, il nous a semblé nécessaire de le prolonger par une mise en perspective. Les hommes du Rufiji, aujourd'hui, pour se procurer le revenu monétaire nécessaire à la survie de leur famille, se font braconniers et vont pêcher dans les lacs « protégés » de la Réserve du Selous. Or naguère ces lacs étaient les lieux de pêche de leurs pères, leurs alentours les lieux de résidence, de culture et de cueillette de leurs familles. Dans l'histoire de l'expropriation des communautés du Rufiji de ces lieux-là, les politiques successives de gestion des ressources naturelles et de la faune sauvage occupent une place centrale. Nous en faisons un bref rappel critique pour nous attarder sur leur dernier avatar, la politique de Community-Based Natural Resources Management (CBNRM, qualifiée aussi de Community-Based Conservation, CBC). Les défenseurs de la politique du CBNRM la présentent comme la meilleure réponse à l'incapacité de l'État et du Marché à promouvoir une gestion durable et équitable des ressources naturelles dans les pays en « développement $»^{3}$; elle améliorerait la préservation de ces ressources par l'implication des communautés rurales concernées dans leur gestion et par un partage plus équitable des coûts et des bénéfices induits (Li 2002). Comme Li le souligne, la reconnaissance du concept de CBNRM dans les politiques nationales et son inscription dans le cadre juridique sont indéniables, mais son impact réel sur la vie des populations doit être mesuré (ibidem). Nous montrons que, dans le Rufiji, en dépit du fait qu'à certaines échelles les projets de conservation ont pu contribuer au « développement » (Kareiva et al. 2008), cet impact est extrêmement faible et témoigne, ici comme ailleurs, du « fossé croissant entre le discours et la réalité » (Igoe et Brockington 2007 : 442).

Pour clore cette introduction, il nous faut préciser que la question des populations vivant à proximité immédiate ou au sein d'aires protégées s'inscrit dans une problématique plus large,

2. Le premier auteur a séjourné dans ce village de novembre 2006 à septembre 2008 (soit une fois soustraits les congés et les séjours à la capitale, environ 14 mois), un séjour complété par une mission en juillet, août et septembre 2009.

3. Les guillemets sont ici pour rappeler l'imposture que recouvre ce mot de développement (voir notamment Rist 2001). 
celle des modes d'appropriation et de mise en valeur des ressources environnementales comme élément décisif de la reproduction des communautés paysannes. À ce titre les politiques de conservation de la nature ne sont qu'une des facettes d'une tragédie multiforme dont le Rufiji est le théâtre. Les communautés paysannes y sont menacées d'une restriction sans précédent de leur accès aux ressources du milieu par un véritable déferlement de grands projets de « développement » qui inclut notamment la construction d'un grand barrage hydroélectrique (Duvail et Hamerlynck 2007) et l'aliénation de milliers d'hectares pour la production de biofuels (Duvail, Médard et Paul 2010). Les forêts villageoises du Rufiji ont quant à elles été dépouillées depuis plusieurs années de toutes les essences de valeur commerciale (Ahrends et al. 2010) au profit exclusif de quelques hommes d'affaires et d'une partie de la bureaucratie et de l'appareil politique tanzaniens.

\section{Braconner pour survivre}

Hassan est un homme heureux, heureux et anxieux à la fois. Ce qu'on appelle des sentiments mêlés. Il est heureux parce que sa fille vient de réussir l'examen d'entrée à l'école secondaire et que son fils est très bien placé sur la liste complémentaire. Deux enfants d'une même famille admis à l'école secondaire la même année, c'est un événement dans ce village. Un village d'un district réputé « sous-développé » au sein d'une Tanzanie elle-même classée parmi les pays les moins avancés ${ }^{4}$. Il est anxieux parce qu'il se demande comment réunir l'argent nécessaire à la scolarisation de ses deux enfants. Il sait que les années d'études secondaires sont une véritable pénitence pour les parents. Les calculs sont vite faits. La première année est la plus coûteuse : il faut investir dans un matelas, une tenue de sport, des chaussures de ville, un seau, et d'autres choses encore. Il y a aussi les droits de scolarité, le matériel scolaire, l'internat, la cantine... Au bas mot, lui a dit Sultani dont les trois fils sont en secondaire, pour chacun de ses enfants, il lui faudra débourser 300 euros pour l'année, une véritable fortune.

Hassan n'a pas beaucoup été à l'école, mais il sait lire et compter. Compter surtout. Comme la plupart des autres villageois, sa seule source de revenu fiable et régulière, c'est la pêche. Il a bien une trentaine d'anacardiers mais la récolte de la noix de cajou n'a lieu qu'une fois l'an et il suffit d'une forte pluie à l'époque de la floraison ou d'un feu de brousse qui gagne sa plantation avant le désherbage pour réduire à néant tous les espoirs de revenu. Et surtout, même si la récolte est bonne cette année, qu'en sera-t-il du prix et du ramassage par la coopérative encore naguère moribonde ? L'an passé la coopérative n'avait pas assez de fonds pour acquérir la récolte de tous les villageois, seuls ceux à jour de cotisation ont pu tout écouler. Pour les autres, les acheteurs privés sont venus et ont tout acheté à bas prix. L'année précédente avait été pire encore. Beaucoup n'avaient pas trouvé d'acheteurs et leurs noix avaient pourri dans les sacs ${ }^{5}$.

4. En 2004, le PIB/habitant de la Tanzanie est classé $225^{\mathrm{e}}$ sur 232 pays et s'élève à 700 US\$ (http://www. cosmovisions.com/RangPIBparHabitant.htm). En 1996, seulement 7\% de la classe d'âge concernée réussissaient l'examen d'entrée en école secondaire (Rufiji District : 1997).

5. Le rendement moyen en Tanzanie est estimé à $3 \mathrm{~kg}$ de noix non décortiquées par pied (Azam-Ali et Judge 2001). Au village d'Hassan, en décembre 2006, la coopérative a acheté les noix non décortiquées pour 0,21 euros $/ \mathrm{kg}$. L'estimation du revenu annuel d'Hassan pour sa trentaine d'anacardiers est d'environ 19 euros. 
Tableau 1

Estimation des coûts de scolarisation d'un élève de première année du secondaire.

\begin{tabular}{|l|l|}
\hline Item & Valeur en $€$ \\
\hline Nourriture & 202,5 \\
\hline Pétrole lampant & 30,24 \\
\hline Vêtements & 22,8 \\
\hline Frais de scolarité & 25,8 \\
\hline Fournitures & 11,4 \\
\hline Loyer & 5,4 \\
\hline Matelas & 7,8 \\
\hline Déplacement & 7,2 \\
\hline Total & $\mathbf{3 1 3 , 1 4}$ \\
\hline
\end{tabular}

Source : enquête auprès de Sultani, village d'Hassan district du Rufiji, Tanzanie, novembre 2007.

Il y a aussi le sésame. Depuis l'année dernière, il a un bon prix, au moins 0,42 euros $/ \mathrm{kg}$. C'est la maison d'export Fida Hussein qui l'achète pour fournir les marchés bio européens. Mais cette année ? Et si le temps est mauvais, qu'il pleut à la floraison. Et si, et si ${ }^{6} \ldots$ La seule source de revenu fiable et régulière est donc la pêche. La demande citadine jamais satisfaite et la concurrence entre les acheteurs assurent la stabilité du prix du poisson et il n'y a pas d'invendu.

Hassan, comme la plupart des hommes du village, pêche le plus souvent dans l'un des plus grands lacs de la vallée sur le rivage duquel le village a été installé lors de la villagisation de 1967. Embarqués sur une pirogue monoxyle avec son compère Salum, ils posent leur filet le soir et le relèvent au petit matin, dormant sur la berge pour décourager les larcins. Une simple sieste effacera la fatigue d'une nuit à la dure et il leur sera possible d'occuper leur journée à d'autres activités, souvent agricoles. Suivant la saison, il leur arrive également de pêcher, de jour ou de nuit, à la senne simple embarquée (kuchokoa) et, dans ce cas, ils seront trop épuisés pour accomplir quelque autre travail durant la journée. Plus rarement, Hassan abandonne le lac pour le fleuve où il pose des palangres (ndowano ya kutega). C'est le cas lorsque les conditions de pêche dans le lac sont défavorables, ou quand les travaux agricoles l'obligent à rester dans la plaine inondable, sur la rive du fleuve opposée au lac.

Bon an mal an, cette pêche de proximité ${ }^{7}$ lui rapporte en moyenne, estime-t-il, 1,2 euros par jour. S'il pêchait tous les jours, il pourrait espérer un revenu annuel de 438 euros. Il lui faut 6. Le rendement moyen du sésame en Tanzanie est de $300 \mathrm{~kg} / \mathrm{ha}$ (FARN 2007). Hassan estime sa surface à
0,2 ha soit un revenu annuel d'environ 25 euros.

7. Pêche de proximité désigne une pêche, quelle que soit la technique utilisée, qui permet au pêcheur de rentrer quotidiennement au village. 
environ 0,6 euros pour les besoins quotidiens de la famille, soit 219 par an ${ }^{8}$. Resterait 219 euros, ce qui est insuffisant, ne serait-ce que pour la scolarisation d'un seul de ses enfants. De plus, il ne peut pas pêcher tous les jours de l'année. Deux mois durant (entre avril et juin), les eaux du lac sont trop hautes pour permettre une pêche productive, les poissons se réfugient dans la végétation submergée par les eaux où il est impossible de poser ses filets. Même si la plupart du temps l'épouse assure l'essentiel du travail agricole, il y a aussi les journées où la contribution de l'époux est indispensable à la mise en culture des parcelles de maïs et de riz et plus tard à leur récolte, au désherbage de la parcelle d'anacardiers... Il y a enfin les journées occupées par des activités sociales (funérailles, initiation, mariage, réunion de village) et celles perdues en raison de maladie. En fin de compte, pêcher 300 jours par an et obtenir ainsi un revenu annuel de 360 euros serait déjà une prouesse.

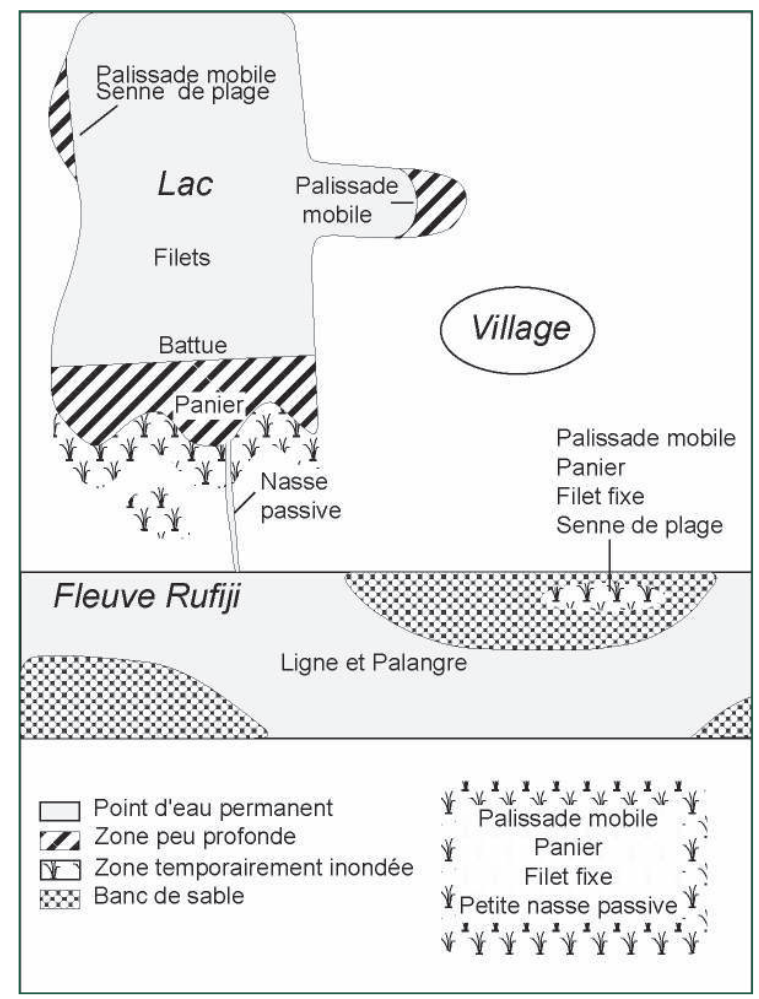

Schéma 1

Schéma du territoire villageois : zones de pêche de proximité et zones cultivées.

8. Les dépenses alimentaires du foyer d'Hassan (en incluant également l'achat du pétrole lampant, des allumettes et du savon) ont fait l'objet d'un suivi quotidien de février 2007 à août 2008. La dépense quotidienne moyenne est de 0,52 euros, moyenne calculée sur 587 jours. Les dépenses « exceptionnelles » ne sont pas incluses (achat de vêtements, frais de scolarisation pour les trois enfants qui sont à l'école primaire, houe, coutelas, vaisselle, cigarettes, soda, etc.). On peut donc considérer l'estimation d'Hassan à 0,60 euros/jour comme raisonnable, voire modeste (source : suivi quotidien 15 janvier 2007 au 31 août 2008). 


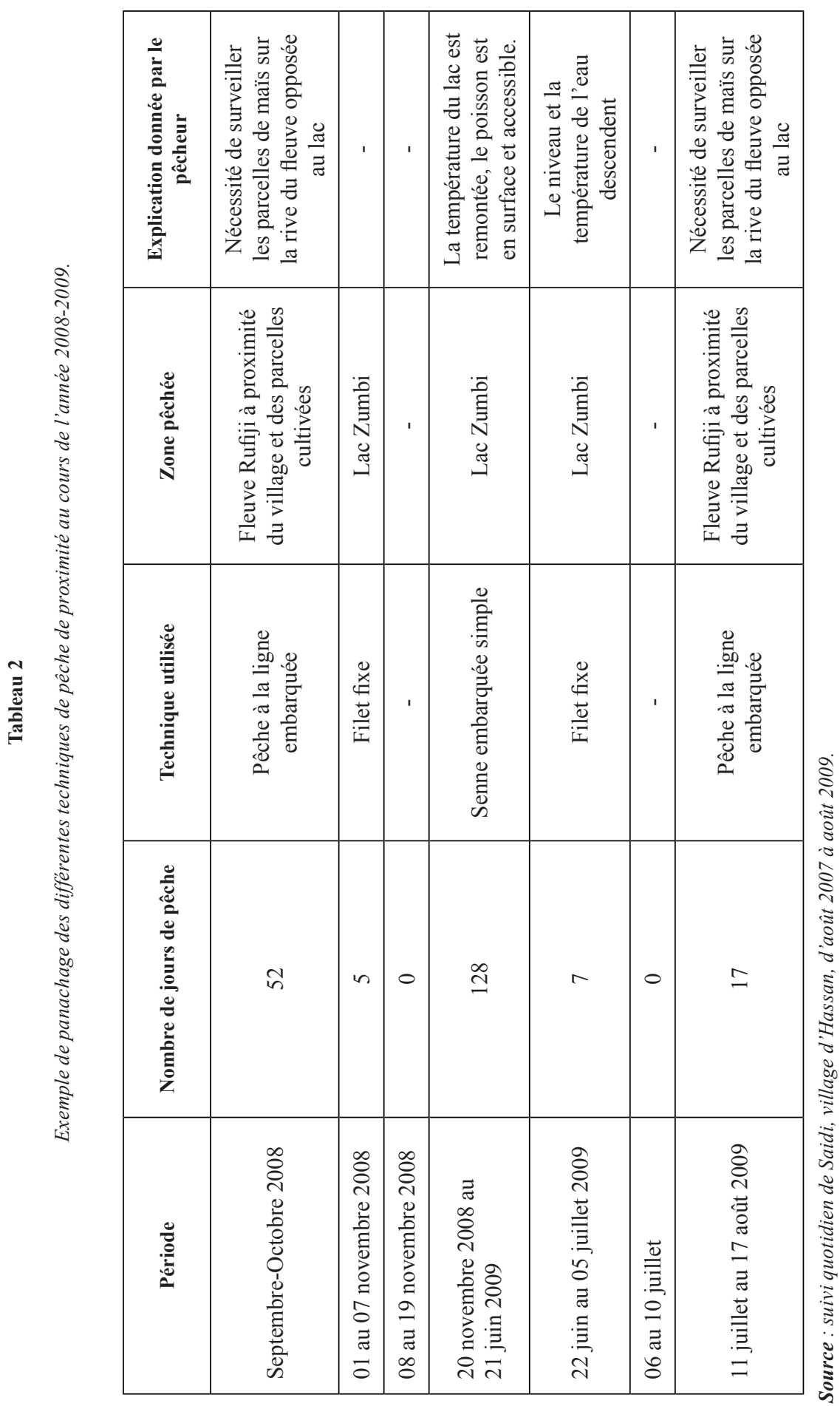




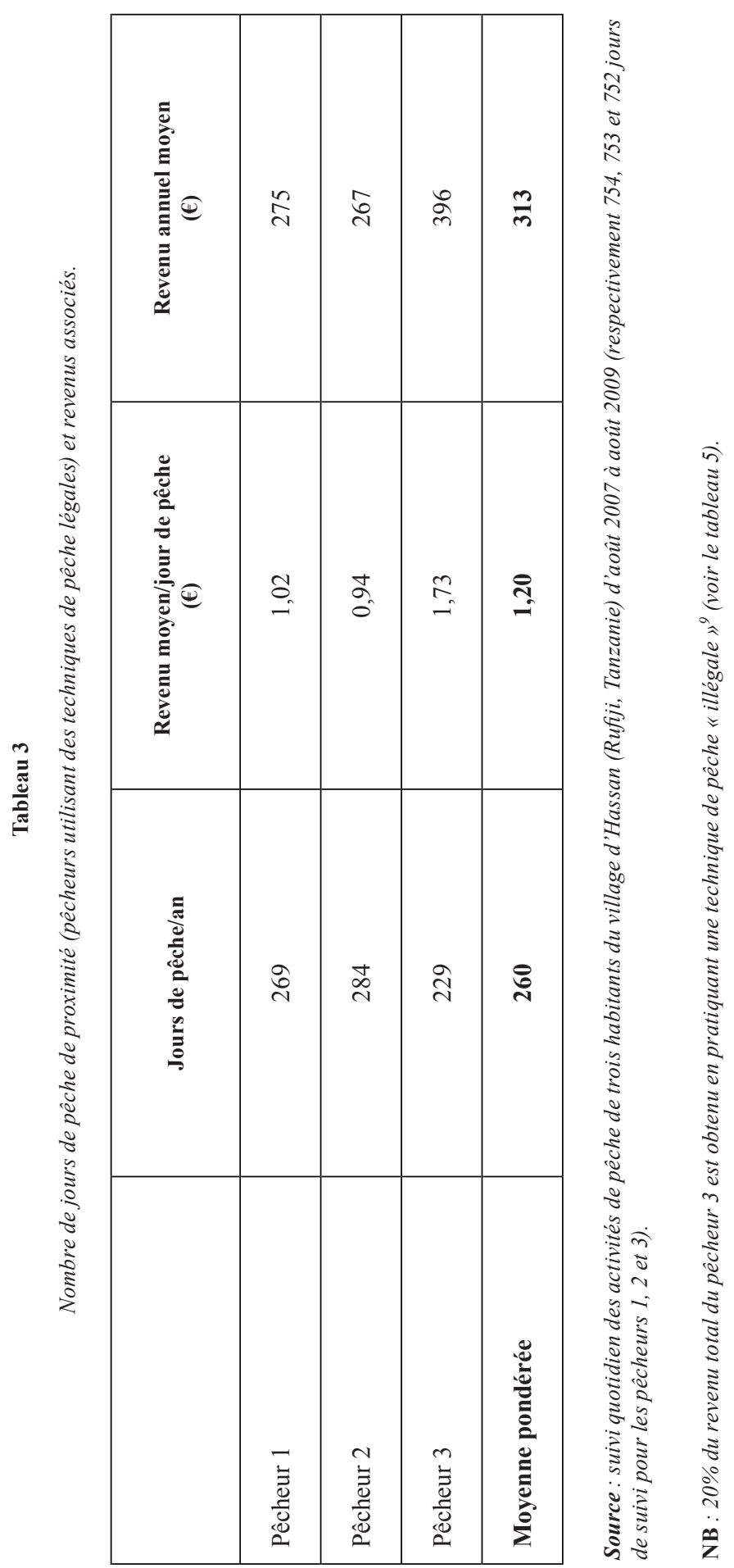




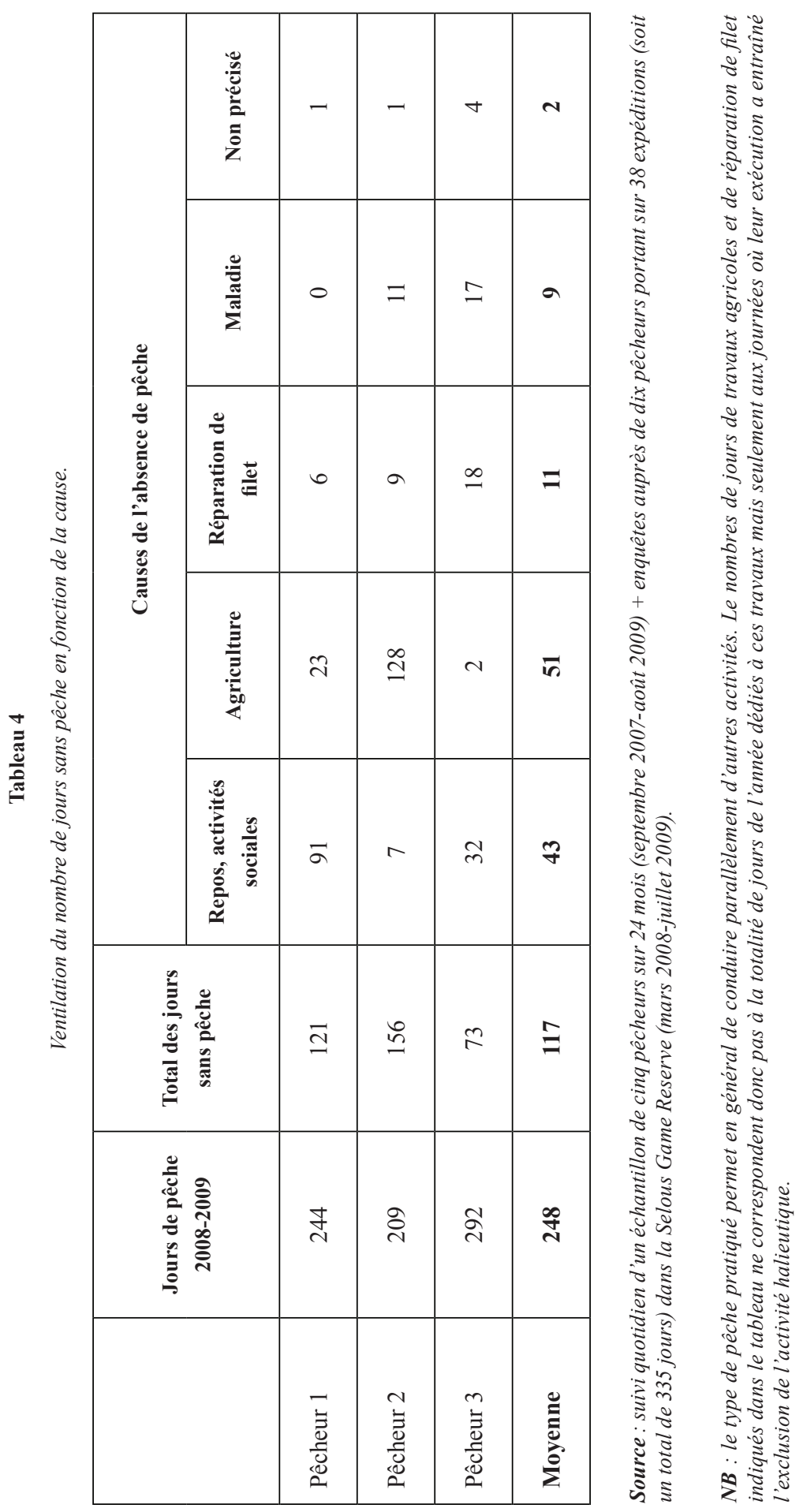


Bien entendu et comme à l'habitude en cas de besoins exceptionnels, on sollicitera la famille. De son lignage ${ }^{10}$, il ne peut guère espérer que l'aide de son frère, c'est-à-dire quelques milliers de shillings. C'est le problème des lignages à faible effectif. Celui de son épouse est plus fourni mais il abrite également plusieurs étudiants et tout ce monde est déjà très sollicité... Même Kimwaga, un membre à part entière de ce lignage, peine à collecter l'argent nécessaire à la scolarisation de sa fille.

En fait, tous ces calculs sont inutiles. La solution, Hassan la connaît, il lui suffit d'observer tous ceux dont les enfants étudient au collège. Ils vont pêcher là où le poisson abonde, là où on peut gagner près de quatre euros par jour d'expédition, trois fois plus qu'au lac du village : dans les lacs poissonneux du «Zaïre ». Le Zaïre, voilà comment on désigne avec humour la Réserve nationale du Selous, $70 \mathrm{~km}$ à l'ouest du village. D'ailleurs naguère, lorsque ses enfants étaient trop jeunes pour participer de manière significative aux travaux champêtres ou domestiques et que les achats à la boutique palliaient souvent l'insuffisance des récoltes, c'était déjà là, au Selous, qu'il allait pêcher. Mais depuis plus de dix ans, ce n'était plus nécessaire. Ainsi, pour réunir les 219 euros nécessaires à un budget familial ascétique et les 600 euros de la scolarisation des deux enfants, il faudrait qu'Hassan pêche environ 200 jours par an au Selous. La pêche dans la réserve est éreintante, on dort très peu. De plus, en saison sèche beaucoup de journées sont perdues en raison de la surveillance accrue des gardes. On ne peut pas envisager plus de 150 jours de pêche par an, soit un revenu espéré de 600 euros. Mis bout à bout avec l'aide des parents, la vente des noix de cajou, du sésame, du coton, de quelques poulets et, si l'année est bonne, des surplus vivriers, Hassan arrivera à ses fins. Inch'Allah ${ }^{11}$.

Tableau 5 ci-après

Revenu moyen (euros) d'un jour de pêche en fonction de la technique et du lieu comparé à la couverture des dépenses annuelles relatives à la scolarisation d'un élève de première année de secondaire.

Source : suivi quotidien d'un échantillon de 5 pêcheurs sur 24 mois (septembre 2007-août 2009) + enquêtes auprès de 10 pêcheurs portant sur 38 expéditions (soit un total de 335 jours) dans la Selous Game Reserve (mars 2008-juillet 2009).

10. Pour éviter l'identification des pêcheurs, ils seront désignés par des prénoms très répandus et leur lignage ne sera pas précisé. Précaution utile ? Igoe, chercheur de l'IUCN rapporte comment lui et son assistant furent menacés d'emprisonnement par le Responsable pour la faune sauvage du district du Rufiji. L'assistant arguant du respect de leurs droits, le Responsable fit cette réponse : «Quand nous arrêtons des personnes, notre tâche n'a rien a voir avec leurs droits. Ceci est une question pour les tribunaux. On vous arrête et plus tard les tribunaux decident si nous avons ou non violé vos droits. Quoi qu'il en soit, entretemps, vous souffrirez un peu" (Igoe 2007 : 245).

11. Le tableau 5 indique qu'il existe deux techniques de pêche de proximité illégales permettant de doubler le revenu par rapport à la pêche de proximité légale. Si Hassan n'envisage pas d'utiliser ces techniques, c'est à la fois parce qu'elles sont particulièrement physiques (seuls les jeunes hommes pratiquent la senne embarquée double) ou techniques (tous les pêcheurs à l'épervier sont étrangers au village) et qu'elles interdisent absolument la conduite parallèle de toute autre activité pour un revenu qui ne représente respectivement que 51 (senne embarquée double) à 63\% (épervier) du revenu de la pêche au Selous. 
Jean-Luc Paul, Stéphanie Duvail \& Olivier Hamerlynck

\begin{tabular}{|c|c|c|c|c|c|c|c|c|c|c|c|c|c|}
\hline 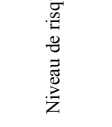 & 鄫 & 盖 & 产 & $\frac{5}{\frac{5}{2}}$ & $\overline{\bar{z}}$ & 善 & 总 & 盖 & & $\frac{\bar{g}}{\frac{0}{2}}$ & & 亳 & \\
\hline 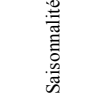 & 惫 & $\frac{0}{\frac{0}{2}}$ & 善 & $\begin{array}{l}\text { 产 } \\
\frac{2}{2} \\
\frac{2}{2}\end{array}$ & 善 & 営 & 产 & $\begin{array}{l}\frac{0}{2} \\
\frac{2}{2}\end{array}$ & & 总 & & 曾 & \\
\hline 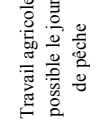 & 亏 & z̃ & 芩 & z̆ & 紊 & 芩 & zू & $\bar{\prime}$ & & ฉू & & zू & \\
\hline 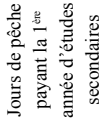 & ळે & 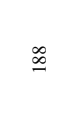 & ปे & $\stackrel{9}{=}$ & $\stackrel{\infty}{\sim}$ & $\stackrel{\circ}{\dddot{2}}$ & \multicolumn{2}{|c|}{ 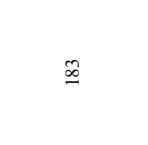 } & & $\stackrel{\infty}{\circ}$ & & $\therefore$ & \\
\hline 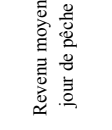 & E. & $\stackrel{8}{-}$ & సे & तु & $\stackrel{0}{=}$ & $\overline{\mathrm{A}}$ & \multicolumn{2}{|c|}{$\stackrel{t}{\sigma}$} & & iे & & $\stackrel{\infty}{\circ}$ & \\
\hline 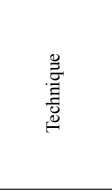 & 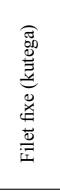 & 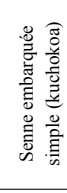 & 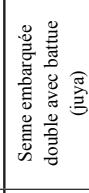 & 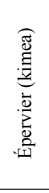 & $\begin{array}{l}\text { 垔 } \\
\text { 产 } \\
\text { 言 } \\
\text { a }\end{array}$ & 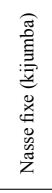 & 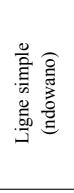 & 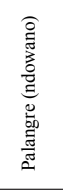 & & 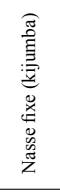 & & 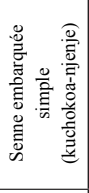 & \\
\hline 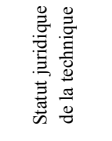 & \multicolumn{2}{|c|}{ 涸 } & \multicolumn{2}{|c|}{ 焣 } & \multicolumn{4}{|c|}{ 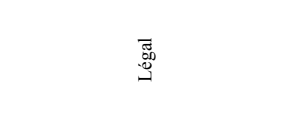 } & 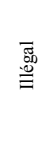 & 骂 & 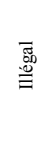 & 㤟 & 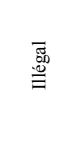 \\
\hline 产 & \multicolumn{4}{|c|}{ 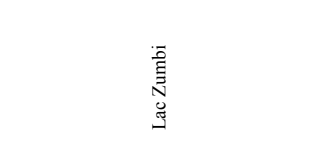 } & \multicolumn{5}{|c|}{ 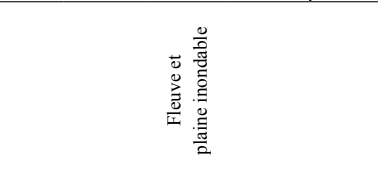 } & \multicolumn{2}{|c|}{ 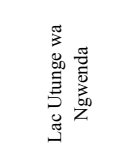 } & \multicolumn{2}{|c|}{ 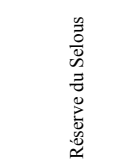 } \\
\hline 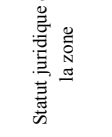 & \multicolumn{9}{|c|}{ 急 } & \multicolumn{2}{|c|}{ 鹗 } & \multicolumn{2}{|c|}{ 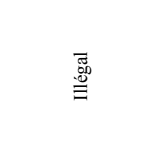 } \\
\hline & \multicolumn{9}{|c|}{ 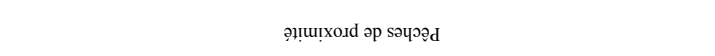 } & \multicolumn{4}{|c|}{ 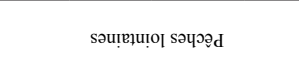 } \\
\hline
\end{tabular}


Voilà comment, à plus de cinquante ans, après avoir abandonné la pêche dans le Selous depuis près de dix ans, Hassan commence en 2007 une nouvelle campagne de pêche, au risque de sa vie, dans l'espoir que ses enfants étudieront avec succès, dans l'espoir que ses enfants un jour auront accès à un travail salarié en ville, dans l'espoir qu'ils ne l'oublieront pas, dans l'espoir d'échapper à une vieillesse misérable, dans l'espoir aussi de rester un homme fier. Il le fait avec détermination, sans ressentiment. Il le fait avec la fierté d'assumer le rôle d'époux et de père, ici et maintenant ${ }^{12}$.

\section{Encart 1 : Récit de Juma, avril 2008 \\ (enregistré et traduit du swahili par le premier auteur)}

Kimwaga, mon père cadet, (...) quand il a appris que sa fille avait réussi l'examen d'entrée en secondaire, il était dans une mauvaise passe (...) Il a montré les résultats à ses proches parents (...) pour qu'ils cotisent (...) On informa également les parents de Dar es Salaam (...) Mon cousin et moi-même et un peu mon père et mon oncle donnèrent chacun 3 euros. Les autres déclarèrent que la situation était difficile, qu'ils n'avaient pas les moyens. Kimwaga ne pouvait plus compter que sur la vente de ses poulets (...) L'argent des noix de cajou avait déjà été utilisé car Kimwaga ne pensait pas que sa fille réussirait à l'examen. (...) Il aurait fallu pour commencer près de 120 euros et seulement 18 euros avait été collectés (...) Le père avait perdu espoir (...) La mère réalisa qu'il fallait qu'elle prenne les choses en main (...) On a décidé qu'elle et sa fille devaient se rendre en ville pour démarcher les parents (...) En les voyant, ils se sentiraient mal à l'aise et cotiseraient (...) Par chance elle collecta là-bas 72 euros (...) De Dar es Salaam, la mère accompagna directement sa fille à l'école (...) Elle y laissa sa fille, ayant payé certaines choses et on lui dit que le reste devrait être payé en juin. Elle revint à la maison et nous raconta (...) À ce moment, j'ai dit moi à mon père cadet : " (..) il faut que tu cherches de l'argent, ne compte pas uniquement sur l'aide de la famille, ce sera insuffisant. Pêche et tu gagneras de l'argent! » (...) Il acquiesça à demi seulement : "Si tu pêches, je t'achèterai les filets qui t'aideront à te faire de l'argent " (...) Je ne l'ai pas revu depuis à ce sujet bien que je le croise presque tous les jours. Il n'a pas réellement la volonté d'aller pêcher. La fille a étudié jusqu'au moment où on l'a renvoyée parce qu'elle n'avait pas payé. Elle est revenue au village pour chercher de l'argent (...) Il n'y avait pas d'autre solution que de vendre le paddy que

12. Au sein des populations du Rufiji, la division sociale du travail assigne à l'époux la responsabilité des dépenses monétaires du foyer. Le suivi des dépenses quotidiennes de neuf foyers durant deux ans et demi montre qu'effectivement l'époux couvre en moyenne $70 \%$ des dépenses (contre $20 \%$ pour la ou les épouses, les autres membres du foyer couvrant le reliquat). Les revenus monétaires des hommes proviennent en quasi-totalité de la pêche. Lors de discussions entre hommes au camp de pêche, nous avons constaté à plusieurs reprises la relation qu'ils établissent entre la disponibilité sexuelle de leur épouse et leur capacité à couvrir les besoins monétaires du foyer. 
la mère avait récolté (...) Ils calculèrent qu'ils vendraient le paddy puis viendrait la récolte des noix de cajou et ensuite celle du coton Ils conclurent que la vente de ces cultures pouvait couvrir les frais de scolarisation (...) Malheureusement, la rentrée scolaire arriva et le prix du paddy restait bas (...) Ils voulurent m'emprunter 42 euros. «Je n'ai pas les moyens de vous prêter une telle somme en ce moment. Allez vendre votre riz comme vous l'aviez prévu ». La mère tenta d'autres démarches que je ne connais pas car après mon refus elle était fâchée (...) et sa fille est repartie au lycée avec seulement 18 euros. Arrivée là-bas (...) on l'a renvoyée de nouveau. Elle revint au village et il fallut chercher de l'argent encore (...) Ils vendirent des poulets et des noix de cajou (...) Elle prit l'argent, retourna à l'école. Elle trouva les élèves en examen de fin d'année. Elle passa simplement deux des épreuves au lieu des dix prévues et, après une semaine, elle rentra chez elle (... $)^{13}$. De retour au village, il fallait songer à lui procurer à nouveau de l'argent (...) Quand la mère expliquait la nécessité de chercher de l'argent son époux se faisait sévère et ne la soutenait pas. "Je n'ai pas d'argent (...) qu'elle reste à la maison! » La mère n'était pas du tout d'accord. "Si tu ne trouves pas d'argent ici va pêcher au Selous! Là-bas il y a la possibilité d'obtenir rapidement de l'argent» (...) Un jour il vint à la maison pour me dire : "Ma fille a besoin de 42 euros (...) J'ai l'intention d'aller pêcher au Selous. Qu'est-ce que tu en penses?" "Si tu as décidé d'aller au Selous, c'est bien. Qu'attends-tu de moi ? " Quand je serai prêt je te le dirai, peut-être le filet ou une pirogue. J'irai au Selous avec ton frère Mudi. Si je dois mourir, je mourrai là-bas! »Il semblait décidé mais finalement il changea d'avis. Ils cherchèrent de l'argent ici et là, ils vendirent des poulets, quelques mangues. Finalement l'argent suffit pour que l'enfant reparte à l'école. Mais son épouse veut l'obliger à aller pêcher au Selous, pour chercher de l'argent et l'aider.

\section{Risquer sa vie pour une poignée de dollars...}

La Selous Game Reserve se trouve à environ 70 kilomètres du village. À de rares exceptions près, les pêcheurs se rendent en autobus ou à bicyclette jusqu'au village de Mloka, situé en bordure de la réserve, où ils retrouvent les pêcheurs venus d'autres villages. Le village d'Hassan est le plus éloigné du Selous où un nombre significatif de villageois pêchent dans le Selous. Au-delà, il semble que la distance et la diversité des sources de revenu monétaire (liée à la proximité relative des centres urbains de Kibiti, d'Utete et d'Ikwiriri) rendent la pêche dans le Selous peu attractive ${ }^{14}$. Avant la dernière extension orientale de la réserve à la

13. Le passage en seconde année est automatique, le passage en troisième année nécessitera la validation des huit examens manquants.

14. Il faut aussi souligner qu'il existe un gradient pluviométrique rapidement croissant vers l'est (influence maritime). Les résultats de l'activité agricole sont donc moins aléatoires dans les villages à l'est du village d'Hassan. 
fin des années 1960, l'activité halieutique y était techniquement diversifiée et concernait des milieux variés : le fleuve (notamment aux abords des rapides de Stiegler's Gorge), les mares temporaires et les lacs permanents. Désormais, seuls ces derniers, au nombre de sept, sont concernés ${ }^{15}$ et, comme nous le préciserons plus loin, une unique technique est utilisée. En raison de la protection dont ils sont l'objet, ces lacs sont restés poissonneux.

L'équipage est constitué de deux hommes embarqués sur une pirogue monoxyle et est relativement stable d'une expédition à l'autre. Souvent plusieurs pirogues débutent ensemble l'expédition puis se répartissent éventuellement sur plusieurs lacs où elles retrouveront d'autres embarcations les ayant devancées. Se regrouper réduit certains risques : on pourra être secouru en cas de problème, emprunter un filet si le sien est prématurément déchiré, etc. Mais, plus nombreux, on est aussi plus aisément repérables. C'est ainsi que quelques-uns, en général de vieux briscards ou des têtes brûlées, préfèrent naviguer en simple paire afin de rester plus discrets.

La pêche dans la Réserve du Selous est très contraignante à différents points de vue et présente plusieurs particularités. La première de ses caractéristiques est le recours à une sorte "d'armateur ». Lorsqu'il pêche à proximité du village, le revenu obtenu par le pêcheur couvre tout juste les besoins de la famille au jour le jour. S'il rentre bredouille ou s'il s'attarde, il est fréquent que, dans l'incapacité de se procurer de la farine de maïs, un accompagnement ou d'autres ingrédients, l'épouse supprime le repas de la mi-journée. L'absence de trésorerie interdit donc au pêcheur l'éloignement du foyer. Or une expédition au Selous dure en moyenne neuf jours ${ }^{16}$. Le pêcheur est de même incapable d'auto-financer la nourriture et le matériel nécessaires à l'expédition. Le filet, par exemple, coûte une dizaine de milliers de shilling (six euros) soit l'équivalent du revenu de cinq jours de pêche de proximité. Rendu inutilisable par les conditions de pêche, il sera abandonné dans le Selous au retour de l'expédition. À l'occasion d'une expédition de plusieurs jours ${ }^{17}$, l'acheteur de poissons se transforme donc en armateur et fait une série d'avances :

- une somme remise à la famille et qui lui permettra de subsister le temps de l'expédition ;

- la location de la pirogue, généralement une pirogue bon marché, de bois tendre, étant donné les importants risques de perte ;

- l'achat du matériel dont l'élément principal est le filet. Le plus souvent le filet, déchiré à plusieurs reprises par les crocodiles, les hippopotames ou les souches immergées, sera abandonné sur le lieu de pêche. S'ajoutent généralement au filet : des piles pour une lampe torche, des allumettes, un bâche plastique pour protéger le poisson fumé durant le voyage retour et quelquefois des cigarettes;

- la farine de maïs et le sel qui serviront à la préparation des repas évidemment accommodés avec du poisson.

15. Les autres anciens lieux de pêche n'autorisent pas la pêche nocturne et discrète qu'impose la surveillance des gardes du Selous (voir infra).

16. Moyenne calculée à partir d'enquêtes auprès de dix pêcheurs et portant sur trente-huit expéditions totalisant 335 jours.

17. Outre le Selous, il existe un autre lieu de pêche distante, le lac Utunge wa Ngwenda dans la plaine inondable, à six à douze heures de marche du village suivant la saison. La pêche y est légale (voir tableau 5). 


\section{Carte 1}

Les lacs de la partie nord-est de la Réserve du Selous.

(C) Stéphanie Duvail.

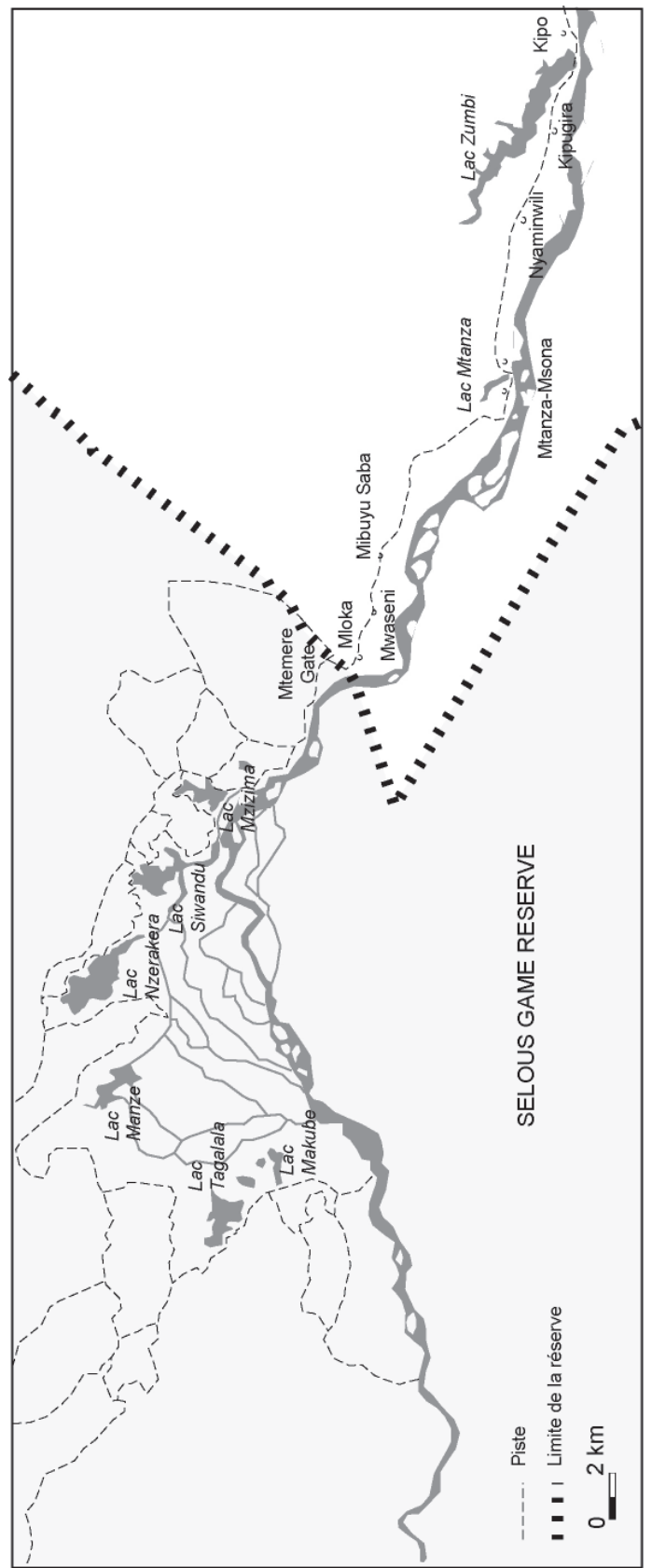




\section{Tableau 6}

Avance (et sa ventilation) faite par l'armateur à deux pêcheurs à l'occasion d'une expédition de pêche de neuf jours dans l'un des lacs du Selous.

\begin{tabular}{|c|c|c|c|c|c|c|c|}
\hline Item & Filet & $\begin{array}{c}\text { Location } \\
\text { de pirogue }\end{array}$ & $\begin{array}{c}\text { Bâche } \\
\text { plastique }\end{array}$ & $\begin{array}{c}\text { Farine et } \\
\text { sel }\end{array}$ & Divers & $\begin{array}{c}\text { Total de } \\
\text { l'avance } \\
\text { monétaire } \\
\text { aux deux } \\
\text { familles }\end{array}$ & Total \\
\hline Coût $(€))$ & 6 & 6 & 1,8 & 4,95 & 2,28 & 12 & $\mathbf{3 3 , 0 3}$ \\
\hline
\end{tabular}

Source : enquête août 2009.

$\mathrm{Au}$ retour, le poisson fumé sera exclusivement vendu à l'armateur qui recouvrera alors les avances consenties. Celui-ci supporte le risque financier (tandis que les pêcheurs risquent simplement leur vie...). Si l'embarcation des pêcheurs est détruite par un hippopotame ou si les gardes saisissent la cargaison au retour, la dette des pêcheurs sera annulée. Pour l'armateur dont le capital permet en général le financement parallèle de plusieurs expéditions, ces expéditions sont synonymes d'un approvisionnement important et régulier.

La seconde des caractéristiques de la pêche au Selous est qu'elle est strictement nocturne. Comme toutes celles des administrations du pays qui ont une fonction répressive, les gardes du Selous ont hérité du passé colonial des pratiques particulièrement violentes. Ils n’hésitent pas à mettre en danger la vie des pêcheurs voire à les abattre dans des situations qui ne le justifient pas. Le premier objectif d'un pêcheur sera d'éviter absolument de les rencontrer. Toutes ses activités seront donc nocturnes.

\section{Encart 2 : Récit de Shamte, avril 2007}

(enregistré et traduit du swahili par le premier auteur)

Je suis allé pêcher au Selous après avoir vu qu'il n'y avait pas de poisson au lac Zumbi (près du village). Il fallait que je trouve un autre moyen d'attraper du poisson. J'ai pêché dans de nombreux lacs $d u$ Selous (...) J'ai été là-bas pour voler du poisson, pour que ma vie soit meilleure. Je m'y suis rendu 33 fois et la dernière fois je me suis fait poursuivre par les gardes (...) On pêchait à Utunge (...) Il faut quatre jours à pagayer du village jusqu'à Utunge. Il faut se déplacer la nuit, le jour c'est impossible. (...) Une fois arrivés à Utunge on s'est mis à pêcher de nuit. Mais on restait bredouilles à cause des crocodiles. Alors on s'est mis à pêcher durant la journée. Le premier jour, on a rapporté près de 600 poissons en une seule prise. On est retourné pour les fumer et pour se cacher. Le second jour on est reparti pêcher le matin avec l'intention de retourner à la maison le lendemain. C'est ce matin-là que les gardes nous ont vus (...) Ils se sont dit : 
"voilà des voleurs, allons-y, on va les poursuivre!" Nous, on s'est enfui à pied et par chance tout le monde leur a échappé. On s'est retrouvé la nuit et on a décidé de rentrer à la maison. Ca ne servait plus à rien de pêcher ici. Certains sont rentrés à pied et nous nous sommes rentrés en pirogue avec le poisson que nous avions. Sur le chemin du retour (...) on a croisé des nôtres. Il faut dire que la période était difficile (à cause de la sécheresse). Il ne restait plus de poisson dans les lacs près des villages (...) Donc personne ne manquait de venir pêcher dans le Selous où le poisson est gros et facile à attraper. Avec 20 poissons tu as déjà de quoi faire, ta famille mange pendant toute une semaine! Le drame c'est que l'État, au lieu d'organiser une pêche payante et surveillée a fait de nous des voleurs. (...) On a donc rencontré dix pirogues de "voleurs " comme nous qui remontaient le courant poursuivis par les gardes. Aucune de ces pirogues ne s'en est sortie. Quand j'ai vu ça, j'ai fait demi-tour et je suis rentré dans un bras du fleuve (...) Nous étions 20 pirogues, 2 pêcheurs dans chaque pirogue. Quatorze d'entre elles ont pris une autre direction, nous nous sommes dirigés vers Mzizima. L'armateur était avec nous. On lui a dit : "Il faut que tu ailles chercher du monde pour porter le poisson (...) En moyenne un homme peut porter 80 poissons (...) Comme nous avons plus de 1800 poissons, il faut que tu reviennes avec 18 personnes au moins » (...) L'armateur est parti à pied jusqu'au village. Il est revenu avec 12 personnes. On a préparé les charges mais (...) il $y$ avait plus de charges que d'hommes. On lui a dit : "On t'a demandé d'amener 18 personnes et tu es venu avec 12, tu n'as qu'à porter toimême le surplus de charges!" (...) Nous, nous sommes partis à six. On a marché jusqu'à Mtemere, c'est là qu'on a eu un accident avec les éléphants. Il y avait une mère avec son éléphanteau (...) elle nous a chargé (...) Elle a saisi l'un d'entre nous au visage. Il était blessé au visage et avait reçu un coup de défense dans la cuisse droite et au pied gauche. Il ne pouvait plus marcher. Il a fallu le porter jusqu'au village de Mloka pour qu'on le soigne (...) Moi, j'ai réalisé à ce moment que j'avais failli dire au revoir à la vie. J'ai décidé que je pêcherai désormais au village. Que je gagne 12 ou 18 centimes d'euros, la famille devrait s'en contenter! C'est là que j'ai abandonné la pêche au Selous. Le blessé a été soigné, on l'a emmené jusqu'à Songa puis à l'hôpital de Mwambili. Il a guéri, il est revenu, il a vécu à nouveau dans ce monde. Et puis il s'est dit que puisqu'il avait déjà été blessé là-bas rien ne l'empêchait d'y retourner (...) Il a été emporté par un crocodile, dans le Selous. Son voyage s'est terminé ainsi (...) Au Selous, je n'y serai pas allé sans la faim. Ce qui nous cause du tort ce sont les mois durant lesquels si tu vas pêcher près du village, tu veilles du soir au petit matin pour 12 centimes d'euros (...) Même si tu gagnes 30 centimes, la farine de maïs coûte 18 centimes le kilo et ta famille compte sur toi. C'est comme ça qu'on décide d'aller faire le voleur dans le Selous, pas pour voler des tôles ou autre chose, simplement du poisson! 
La première difficulté est de pénétrer dans la Réserve du Selous. Pendant la crue, le fleuve forme une multitude de bras secondaires et il est relativement aisé de se faufiler nuitamment jusqu'au lieu de pêche. À l'étiage, le lit mineur du fleuve est la seule voie d'accès. C'est aussi la haute saison touristique ${ }^{18}$ : le zèle et l'efficacité des gardes sont à leur paroxysme. À proximité de l'entrée du parc, les gardes ont alors l'habitude d'installer des postes de surveillance dans certains lieux propices. Ces lieux sont connus des pêcheurs. Avant de lancer l'expédition, ils envoient un éclaireur à pied. S'il repère les gardes ou leur canot à moteur amarré le long de la berge, le départ est repoussé, sauf pour les plus téméraires qui tenteront de tromper la vigilance des gardes à l'occasion d'une nuit sans lune. L'opération se répète jusqu'au jour où le poste est temporairement levé. Au retour, le même problème se pose. Comme on descend le courant, la pagaie peut être utilisée comme simple gouvernail, on est donc plus silencieux. Mais la pirogue chargée de poisson est moins maniable et surtout, il n'existe pas de moyen de s'informer de la présence d'un poste de garde ${ }^{19}$.

Une fois à l'intérieur du parc, le voyage continue, toujours en pirogue et de nuit, jusqu'à l'un des nombreux lacs de l'est du Selous. Les pêcheurs choisissent le lac et l'emplacement du camp en fonction de plusieurs facteurs dont les principaux sont l'exposition au risque (faune sauvage, gardes et propriétaires des camps touristiques ${ }^{20}$, possibilité de camouflage), la densité en poissons et la facilité d'obtention du bois de fumage.

Ensuite, toutes les activités restent nocturnes, évitant même les nuits de pleine lune : la pêche elle-même, la collecte du bois et le fumage du poisson. On ramassera le bois de fumage et on fumera le poisson avant l'aube pour éviter que la fumée ne signale sa présence. On installera son campement dans des fourrés éloignés de la berge, on immergera sa pirogue durant la journée. La pose de filets fixes n'est pas possible, car non seulement les gardes les repéreraient mais encore ils seraient rapidement mis en pièces par des crocodiles voraces. La technique de pêche utilisée est la senne simple (kuchokoa ou njenje). Elle nécessite la coopération de deux pêcheurs embarqués dans la même pirogue. Les pêcheurs décrivent un cercle en mettant le filet à l'eau, une fois le cercle fermé, ils tirent le filet en extrayant les poissons au fur et à mesure de la levée. L'opération est ainsi répétée jusqu'aux environs de 3 heures du matin. On s'interrompt alors pour collecter du bois et fumer le poisson avant que le jour ne soit complètement levé. Le fumage sera repris vers 18 heures avant de repartir à la pêche ${ }^{21}$. On pêche en moyenne une huitaine de jours. C'est le temps estimé pour atteindre la

18. En saison sèche, les pistes de la réserve sont toutes carrossables et les animaux se concentrent autour des points d'eau. Il est plus aisé de satisfaire les exigences des clients. À la saison des pluies, beaucoup de camps touristiques sont fermés.

19. Depuis la fin 2008, l'installation d'une antenne téléphonique non loin de la limite orientale de la réserve permet aux pêcheurs de capter les messages (texto) sur un téléphone portable. Certains d'entre eux sont ainsi informés, sur le lieu de pêche, de la présence et de l'emplacement d'un poste de garde à la sortie du parc. On peut se procurer un téléphone portable pour 5 euros environ, un numéro gratuitement et les cartes de rechargement les moins chères coûtent 15 centimes d'euros.

20. Le propriétaire d'un camp surplombant l'un des lacs a acquis la réputation de tirer sur les pêcheurs. (source : enquêtes 2008).

21. En raison d'un fumage imparfait, le poisson du Selous se conserve moins bien que le poisson pêché lors des pêches de proximité. On embarque donc quelquefois un adolescent en charge de fumer le poisson pendant que les adultes pêchent. Il surveille également le campement pour chasser les maraudeurs, notamment d'autres pêcheurs et les hyènes. 
capacité de charge de la pirogue. Si la pêche est mauvaise, on réduit la ration quotidienne de farine pour s'attarder quelques jours.

Les lacs du Selous regorgent de crocodiles et d'hippopotames, pour le plus grand plaisir des touristes. Les repérer de nuit est fort difficile. C'est dire qu'en tirant sa senne, on doit s'attendre à trouver un hippopotame irascible ou un crocodile attiré par un repas bon marché. Leur asséner un coup de pagaie ne suffit pas toujours à les écarter. Passer au-dessus d'un hippopotame en plongée n'est pas plus plaisant, il vous jette à l'eau, les sauriens font le reste. Bref, pêcher dans le Selous c'est, au sens propre, risquer sa vie. Et chaque année effectivement quelques pêcheurs disparaissent, qui déchiqueté par les crocodiles, qui broyé par un hippopotame, qui, plus rarement, abattu par les gardes ${ }^{22}$.

Depuis notre première rencontre avec Hassan, plus de deux ans se sont écoulés. Ses deux enfants entament leur troisième année d'études secondaires. Lui continue d'aller pêcher régulièrement dans le Selous bien qu'il évoque quelquefois la possibilité de se lancer dans une activité de substitution, comme un commerce de produits villageois vers la ville. Mais jusqu'à présent il n'a pas réuni la somme nécessaire (une cinquantaine d'euros). De novembre 2007 à août 2009, nous avons pu enregistrer les données relatives à seize des expéditions d'Hassan. Son revenu moyen par jour d'expédition s'élève à 2,81 euros sensiblement inférieur à la moyenne de 3,98 euros (tableau 5). Une expédition a été déficitaire : la cargaison a été saisie par les gardes le neuvième jour alors qu'Hassan était sur le point de sortir de la réserve ${ }^{23}$. À cette occasion Hassan a perdu sa pirogue et tout son matériel, il a essuyé des coups de feu et s'est foulé la cheville en s'enfuyant dans la brousse. Deux autres expéditions de 14 jours chacune n'ont rapporté qu'environ 60 centimes d'euro par jour de pêche. La première fois, Hassan fut surpris à la fin de la première semaine par les gardes, il parvînt à s'enfuir avec sa pirogue et son matériel mais son poisson, encore sur le fumoir, fut détruit. Il changea de lac et reprît la pêche. La seconde fois, au cœur de la saison sèche, la concentration en crocodiles était telle que les filets étaient constamment déchirés et les poissons dévorés.

Pour les premières expéditions, de novembre 2007 à mai 2008, Hassan nous a fait part de l'utilisation de son revenu dans les jours qui suivaient son retour. $21 \%$ directement utilisés pour la famille au village, $36 \%$ pour les lycéens, $43 \%$ dépensés plus tard pour des destinations diverses mais qui concernent essentiellement ces deux mêmes catégories (tableau 7).

22. Sur une période de 18 mois, les accidents corporels importants et décès dans le Selous dont nous avons été incidemment informés et que nous avons pu vérifier sont les suivants : un pêcheur a dû être amputé d'une jambe à la suite de l'attaque d'un hippopotame, un pêcheur aurait été tué par balle par les gardes (ses compères ont fui et le cadavre n'a pas été retrouvé), un pêcheur tué par balle par les gardes (le cadavre a été récupéré), un pêcheur tombé à l'eau et dévoré par les crocodiles.

23. Du point de vue purement comptable, il n'y a pas de déficit. En effet, lorsque la cargaison de poissons est saisie par les gardes ou jetée à l'eau par un hippopotame, l'armateur supporte les pertes. Pour le pêcheur, le revenu se trouve ainsi réduit à l'avance faite à la famille et, éventuellement, à l'avance pour l'achat de farine et de sel consommés au cours de l'expédition, soit en moyenne un maximum de 8,5 euros (voir tableau 6). 


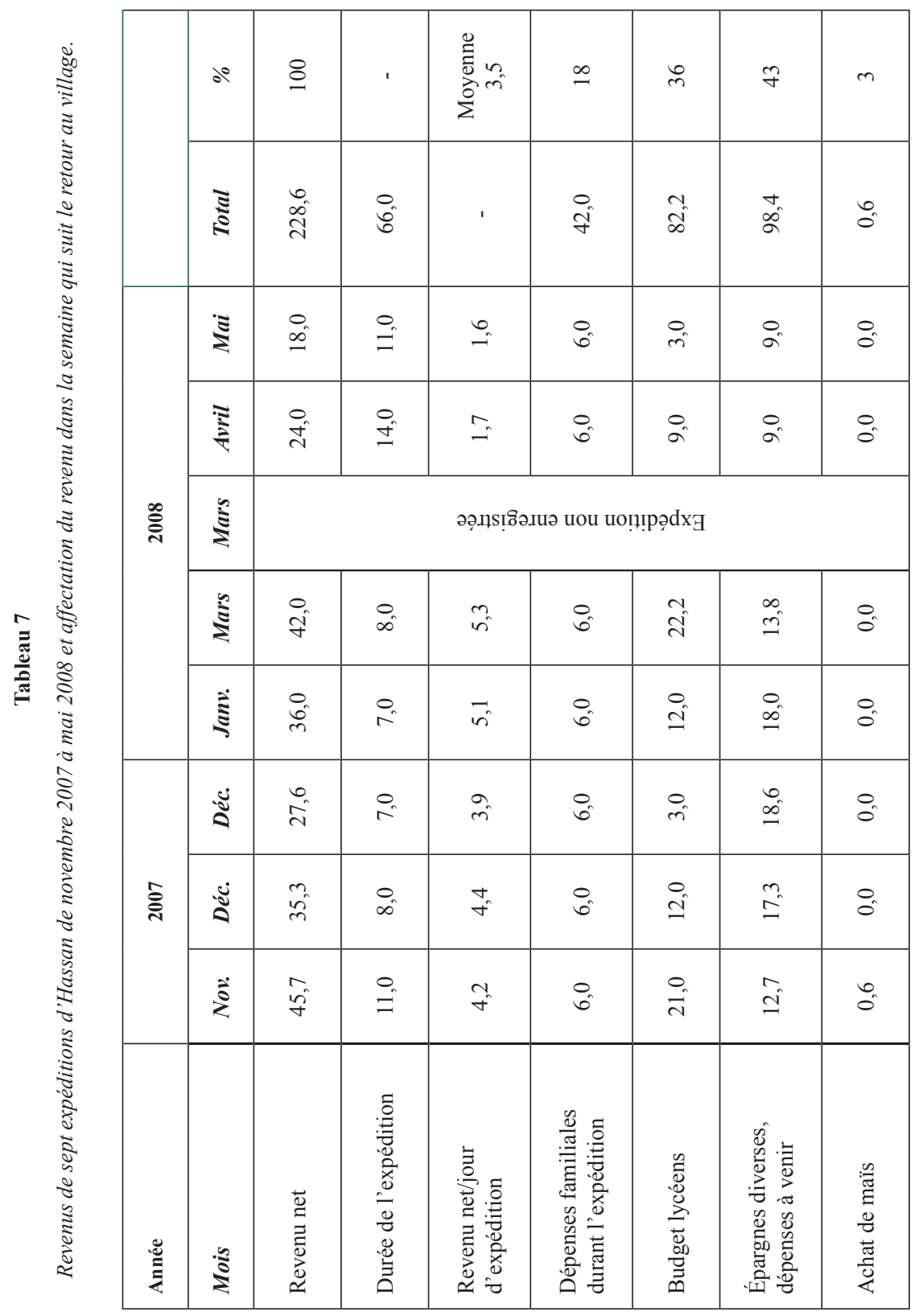


Saidi a le même âge qu'Hassan. Un an après lui, en 2008, son fils a réussi l'examen d'entrée à l'école secondaire. La première année, il a réussi à la financer tant bien que mal par des moyens que nous ne connaissons pas. Mais, à la fin de l'année 2009, il a dû se résoudre à retourner au Selous. Il avait abandonné la pêche au Selous depuis la fin des années 1980. À cette époque, il avait été malade et n'avait pas pu participer à une certaine expédition. Son armateur, un homme du village, avait besoin de poisson pour faire tourner son capital. Il était donc parti à la place de Saidi avec son partenaire habituel. Il a été capturé par les gardes qui l'ont sévèrement battu. En le voyant revenir à moitié mort de la prison du district, après avoir payé une lourde amende, Saidi décida de ne plus jamais remettre les pieds au Selous. Et pourtant... Saidi n'a pas eu la chance d'Hassan. Dès sa première expédition, en novembre 2009, les gardes l'ont capturé. C'était à l'occasion d'une opération spéciale de lutte contre le braconnage ${ }^{24}$. Des douze pêcheurs du camp, il fut le seul à être capturé sur place. Il s'était assoupi et n'a pu s'enfuir avec ses comparses quand les gardes, armés de leurs fusils, ont envahi le camp. Une prochaine mission nous permettra peut-être de recueillir son témoignage mais nous savons d'ores et déjà qu'il a dû payer 300000 shillings pour être libéré, soit plus de la moitié du coût annuel des études de son fils...

\section{Encart 3 : Récit de Kassim, janvier 2008}

(enregistré et traduit du swahili par le premier auteur)

(...) toute ma vie j'ai élevé mes enfants grâce à cette pêche au Zaïre (Réserve du Selous). J'y allais seulement voler du poisson et rien d'autre! (...) Nous nous étions habitués au lac d'Utunge. Nous atteignirent le lac et nous pêchâmes. Moi j'avais un don, je faisais des rêves prémonitoires. Si je rêvais quelque chose, cette chose arrivait! Un jour j'ai dit à mon compère :

Mbonde, je crois qu'aujourd'hui on va nous attaquer! (...)

Nous avons remonté silencieusement la berge et aussitôt on a vu les gardes qui se faufilaient et regardaient partout. J'ai dit à Mbonde :

Tu as vu le travail?

C'est vrai!

Bon, taisons-nous

24. En mars 2010, le magazine The East-African a révélé les résultats d'une enquête conduite par un panel d'experts nationaux et internationaux. Ils montrent l'implication de responsables administratifs et politiques tanzaniens ainsi que de gardes des parcs et réserves dans un imposant commerce illégal de l'ivoire. 50\% des 11632 tonnes d'ivoire de contrebande saisies en 2009 dans le monde proviennent de Tanzanie et principalement du Selous (origine attestée par l'analyse de l'ADN). L'un des journalistes conclut « Malgré la réalisation d'une opération concertée dans le Selous en novembre 2009, le commerce reste florissant » (Mande 2010 : 6). Quatre mois plus tard, deux tonnes d'ivoire en provenance du Selous sont à nouveau saisies au Vietnam (Mukinda et Muindi 2010). Saidi, comme d'autres simples pêcheurs, a été la victime d'une opération médiatique destinée à rassurer l'opinion et les instances internationales plutôt qu'à mettre un terme à un commerce aussi prospère qu'illégal. 
Les gardes se sont rassemblés, ils étaient trois. Ils sont restés pratiquement une heure à observer sans rien voir. Ca a été comme ça un deuxième puis un troisième jour et puis ils sont partis. On s'est remis au travail et on a pêché l'après-midi beaucoup de poisson. On relevait à chaque fois 500, 600 poissons! Après la pêche on fumait le poisson (...). On fumait le poisson en brousse là où c'était impossible de nous repérer, pas sur la berge (...) Mais la nouvelle génération ne respecte rien. Ils installent leur campement sur la berge, ils étendent leurs filets sur le côté, ils n'ont peur de rien. Ca devient une partie plaisir pour les gardes de surprendre un campement de ce type. J'ai dit à Mbonde :

C'en est fini de la pêche. Qu'est-ce que tu en penses? Tu as vu cette situation? On a élevé nos enfants, ils ont étudié, ils ont fini l'école grâce à la pêche dans le Zaïre. Désormais c'est impossible. C'est trop dangereux (...)

Mbonde m'a répondu :

Moi mon vieux je ne suis pas prêt d'arrêter. Toi tes enfants ont fini d'étudier, les miens pas encore.

(...) C'est ainsi que je suis resté au village, je ne pêche plus du tout. J'ai cherché un autre travail pour avoir de l'argent. Je répare les marmites, les seaux qui sont fendus ou qui ont brûlé. Ca me rapporte un peu d'argent (...)

Les cas d'Hassan et de Saidi illustrent la situation tragique des villageois de la plaine inondable du Rufiji et sans doute de bien d'autres régions rurales tanzaniennes. Cette situation est une fatalité historiquement construite dont les deux aspects les plus saillants sont, en l'occurrence, le coût exorbitant des études secondaires au regard des moyens financiers des villageois et le caractère illégal du moyen le plus indiqué de se procurer l'argent nécessaire. Ce dernier point indique que la description ethno-économique de l'activité halieutique et de sa place dans l'économie familiale s'inscrit dans une problématique plus large, celle des modes d'appropriation et de mise en valeur des ressources environnementales comme élément décisif de la reproduction des communautés paysannes de la région. L'exclusion de droit des communautés paysannes du Rufiji oriental des zones de pêche les plus productives est le résultat d'une longue série de choix politiques qui débute avec la colonisation allemande. Ces choix révèlent les intérêts pluriels (antagonistes parfois, relevant plus souvent d'une collaboration conflictuelle) portés par un large éventail d'institutions appartenant à des niveaux d'organisation politique fort divers et au sein desquels les villageois apparaissent comme quantité négligeable. De fait, l'éviction de droit des habitants du Rufiji oriental des zones les plus poissonneuses n'est certainement pas un objectif en soi des promoteurs de la Réserve du Selous. Il s'agit d'un simple dommage collatéral de la politique de préservation de la faune sauvage. Un dommage collatéral pour les institutions dominantes, une tragédie pour les communautés paysannes dont la reproduction est menacée. Réservant d'autres développements pour de prochaines contributions, nous limiterons donc notre ambition, dans les paragraphes qui suivent, à l'analyse des dynamiques historiques qui ont conduit à l'exclusion des villageois des zones de pêche du Selous. 


\section{L'histoire de la constitution de la Réserve du Selous}

L'histoire de la constitution de la Réserve du Selous débute il y a plus d'un siècle avec la création par les occupants allemands de plusieurs réserves ${ }^{25}$. Jusqu'en 1975, elles seront progressivement étendues, remaniées et consolidées, essentiellement par les occupants britanniques mais également, après l'indépendance, par l'État postcolonial. Aujourd'hui, cet impressionnant « sanctuaire » couvre environ $55000 \mathrm{~km}^{2}$. L'une des forces majeures à l'origine de la création des réserves est le maintien de terrains de chasse au bénéfice exclusif du lobby des chasseurs de gros gibier issus des classes aristocratiques allemande et britannique (Koponen 1994 : 536). C'est dans ce cadre qu'il faut interpréter l'objectif principal affiché par l'administration coloniale britannique pour la création de la Réserve du Selous à savoir, la mise à disposition d'un espace protégé « pour la sauvegarde des éléphants qu'on pensait voir disparaître rapidement » (Matzke 1976:38)26. La prise en compte des intérêts des populations locales est marginale et les décrets successifs pour la protection de la faune (1921, 1940 et 1951) seront avant tout les résultats des ratifications successives de traités internationaux (Majamba 2001 : n.p.). D'autres préoccupations que cynégétiques participeront à la consolidation du Selous notamment un programme d'éradication de la maladie du sommeil en $1950^{27}$. Bien que Matzke présente ce programme comme issu d'une volonté purement sanitaire (Matzke 1976 : 39-41), Kjekshus (1977 : 168-179) et Illife (1979 : 271-272) remarquent avec pertinence que dans le combat contre le parasite au cycle dixène, l'administration choisit d'évacuer les humains des zones infestées plutôt que d'éradiquer le vecteur lui-même, la mouche tsé-tsé, dont la population put au contraire croître dans les zones ainsi abandonnées. Pour Kjekshus, ce choix trahissait une préoccupation qui restera une constante des politiques du pays après l'indépendance : l'intégration des populations dispersées et vivant en marge du mouvement de modernisation dans la dynamique de développement économique du territoire. La rhétorique utilisée à cet effet est désormais connue : en regroupant les populations, on leur offrait l'accès à l'éducation, à la santé, à l'eau courante, au réseau routier, l'appui aux moyens modernes de production agricole et aux conseils techniques. Elles pouvaient à leur tour fournir des produits agricoles pour le marché et/ou vendre leur force de travail, bref se " développer ». La stratégie de concentration de la population proposée par le Provincial Commissioner Rooke Johnston en 1945 (cité par Kjekshus 1977 : 178-179) s’imposera d'ailleurs au gouvernement postcolonial après

25. 1896 : création de deux réserves en Afrique Orientale allemande dont celle de Kisaki au nord de l'actuelle Selous. 1913 : quatorze réserves couvrent un total approximatif de $30000 \mathrm{~km}^{2}$. Trois de ces réserves concernent l'aire actuellement couverte par le Selous (Koponen 1994 : 540). Matzke (1976) offre une description synthétique des différentes étapes de la constitution de la Réserve du Selous. Voir également Matzke (1977) et Rodgers (1976) ainsi que Baldus et al. (2009), notamment pour la période postindépendance. Pour l'ensemble de la Tanzanie voir Nelson et al. (2007).

26. Le commerce d'ivoire vers l'Europe et les États-Unis tout au long du XIX ${ }^{\mathrm{e}}$ siècle, l'épizootie de la peste bovine introduite négligemment par les colonisateurs à la fin du XIX siècle et la chasse aux trophées pratiquée par les Occidentaux sont les principaux responsables de cette raréfaction de l'espèce.

27. Pour Illife, c'est la conjonction des désastres « naturels » (l'introduction involontaire de plusieurs pathologies par les colonisateurs, notamment la peste bovine), des migrations de travailleurs imposées par l'économie coloniale et de la dévastation consécutive à la répression de la guerre anti-coloniale de Maji Maji, qui entraîne la conquête, au début du XX $X^{\mathrm{e}}$ siècle, de larges zones par la mouche tsétsé (Illife 1979 : 163-164, 201202). Le colonisateur résoudrait donc ici un problème qu'il a lui-même créé. 
l'indépendance et accompagnera les dernières étapes de l'agrandissement du Selous entre 1967 et 1974, celles qui concernent le plus directement le village d'Hassan. Ici, la bureaucratie d'État saisit l'opportunité des programmes nationaux de villagisation (Operation Rufiji en 1967-68, Operation Tanzania en 1975) pour annexer à la réserve le delta intérieur du fleuve Rufiji. Les nombreux lacs poissonneux de ce delta, points d'abreuvement de la faune sauvage durant la saison sèche, furent les éléments centraux de la création d'une " petite » réserve naturelle de $2500 \mathrm{~km}^{2}$, dédiée aux safaris-photos et venue s'ajouter à la vaste réserve de chasse. Dans le Rufiji comme dans le reste de la Tanzanie, la villagisation signifiait l'expropriation des droits coutumiers des petites communautés dispersées en hameaux et leur regroupement en villages de quelques milliers d'habitants dans la perspective déclarée d'une meilleure planification du « développement » économique (Sundet 1996 : 61). Les procédures légales correspondant à ces expropriations existaient, mais les politiciens et les bureaucrates considérèrent qu'elles étaient superflues et que l'autorité de l'État faisait loi (Shivji 1998 : 13). À ce titre, non seulement les compensations prévues par la loi ne furent jamais versées ni même évoquées, mais encore, l'État créa un imbroglio juridique que les tentatives d'extinction de droits coutumiers plus tardives n'ont pas réussi à lever (Ibidem ${ }^{28}$. Relocalisées en aval, dans des villages créés sur la rive septentrionnale du fleuve, les anciens habitants du delta intérieur perdirent l'accès légal aux zones de pêche " traditionnelles » désormais gérées par l'administration de la réserve.

Pour conclure le parcours exemplaire que décrit son article, Matzke se fait presque lyrique : « La taille exceptionnelle de la réserve en 1975 est d'autant plus remarquable qu'elle est exempte de droits d'occupation humaine. Tout au long de l'histoire de sa création, de nombreuses forces furent à l'œuvre, pour un résultat toujours égal. La réserve qui s'étendait toujours devint une zone sauvage vide d'hommes, gérée par le gouvernement tanzanien à des fins de protection de la faune sauvage et de développement économique basé sur la faune sauvage » (Matzke 1976 : 41). Parmi les «nombreuses forces à l'œuvre » qu'il évoque, il est clair que les populations locales sont quantité négligeable et de fait, à toutes les étapes de la constitution de la Réserve du Selous et quelles que soient les justifications avancées par l'administration, elles furent déplacées de manière autoritaire et sans négociations préalables ${ }^{29}$. Si l'autoritarisme de l'État tanzanien est généralisé dans le domaine des politiques dites de protection de la faune sauvage, il est renforcé par la vision conservationniste qui considère que la seule solution pour protéger un milieu « naturel » c'est d'en exclure les hommes ou, pour reprendre la formulation de Matzke « la politique de développement séparé des hommes et de la faune sauvage » (Matzke 1971) ${ }^{30}$. Cette option considère a priori et transforme $a$ posteriori les populations locales en ennemis tentant de pénétrer à l'intérieur du territoire défendu considéré comme une forteresse assiégée (Baldus 2009 : 10). Elle atteint son

28. Cet imbroglio ouvrait la porte à des recours de la part des populations expropriées mais ces recours ont été peu nombreux.

29. Pour cette période, Baldus qualifie la politique de protection de la nature «conservation contre les gens » (Baldus $2009: 16$ ).

30. « Développement séparé » (separate development) est l'expression utilisée par le gouvernement sud-africain pour qualifier la politique de création de Bantoustans initiée au début des années cinquante. Cette analogie sans doute inconsciente que fait Matzke nous informe peut-être sur la considération portée aux populations indigènes. 
paroxysme avec l'opération uhai de 1988-1989 à l'occasion de laquelle le Département pour la Faune Sauvage, la Police et l'Armée lancent une opération conjointe de répression du braconnage à l'échelle nationale. Les villageois sont la principale cible de cette opération (Baldus et al. 2009 : 95 ; Hahn et Kaggi 2001 : 44).

L'opération uhai coïncide avec l'avènement de la politique de CBNRM en Tanzanie. Elle est impulsée non par un changement des rapports de force à l'intérieur du pays mais par l'évolution des politiques internationales de protection de la nature et par la pression subséquente des bailleurs de fonds pour leur mise en œuvre par les gouvernements bénéficiaires. Cette évolution se caractérise par le recul de l'approche conservationniste dite de la «forteresse », dont l'opération uhai est une illustration, au profit d'une approche participative. Elle prolonge l'émergence au sein des institutions internationales, à partir des années 1970, d'un nouvel avatar du paradigme du développement, le développement durable ${ }^{31}$. Dans sa phase de maturation, le paradigme du développement durable fut d'abord désigné par le terme d'éco-développement qui avait l'avantage d'exprimer clairement l'idéologie qui le sous-tendait. " Eco » faisait explicitement référence à la fusion des préoccupations économiques et écologiques, environnement et développement étant présentés comme les deux faces d'une même médaille. Dans le domaine de la protection de l'environnement et de la faune sauvage, les politiques « d'articulation entre conservation et développement ont suscité un très fort engouement dans les années 1980 et 1990, avec le déploiement d'un discours participatif dans le champ de la conservation, une multiplication des expériences de gestion intégrée et la recherche d'une synergie entre les politiques de la protection de la biodiversité et des politiques plus larges d'environnement et d'aménagement du territoire » (Jeudi-de-Grissac et Rodary $2009: n p$ ). Avec comme postulat fondateur « que les gens qui vivent à proximité d'une ressource naturelle et en tirent directement leur subsistance ont plus d'intérêt à sa gestion et à son utilisation durable que les autorités de l'État ou des sociétés commerciales lointaines » ( $\mathrm{Li} 2002$ : 265), le CBNRM est la forme institutionnelle dominante de ces approches participatives.

Dans le Selous, le CBNRM sera portée par l'agence allemande pour la coopération technique (GTZ) qui administrera la réserve conjointement avec le Tanzanian Wildlife Division dans le cadre du Selous Conservation Programme (Ndunguru et Hahn 1998 : 2-3 ; Baldus et al. 2009 : 95). L'idée est « d'établir, en coopération avec les communautés locales, un programme d'utilisation durable de la faune sauvage dans les zones tampons de la Réserve de Faune du Selous (Ndunguru et Hahn 1998 : 3). À terme, la zone tampon devrait former une ceinture continue à la périphérie du Selous. En 2000, les premières expériences menées au nord, à l'est et au sud de la réserve sur cinq aires discontinues regroupaient quarante et un villages. Les villages de la plaine du Rufiji adjacents à la réserve, dont le village de Hassan fait partie, devraient bientôt bénéficier d'une extension de la zone tampon grâce aux efforts de la coopération belge ${ }^{32}$ et la création de Wildlife Management Areas (WMA) est prévue pour les toutes prochaines années. Pour l'heure, on peut se baser sur les résultats obtenus dans les zones où des WMA sont déjà fonctionnelles pour mesurer l'impact de la nouvelle politique participative tanzanienne de préservation de la faune sauvage et se demander si

31. Pour une critique synthétique de l'oxymore « développement durable », voir Latouche 2001.

32. Dans le cadre de l'Eastern Selous Community Wildlife and Natural Resources Management Project, la Belgique coopère avec la Tanzanie en prolongeant les travaux conduits dans les années 1990 par l'UICN. 
elle est à même de changer la situation des villageois. Pragmatiquement, est-ce que cette politique permettrait à Hassan, Saidi et à leurs compères de ne plus risquer leur vie pour payer les études de leurs enfants ? Les revenus issus de la mise en place d'une WMA dans leur village permettraient-ils de se passer des revenus de la pêche illégale dans le Selous ?

\section{Premier bilan du CBNRM à l'échelle des communautés rurales à proximité du Rufiji : une goutte d'eau dans le désert}

À l'occasion d'un atelier international sur les CBNRM, Ndunguru et Hahn (1998) présentèrent un papier intitulé avec optimisme "Réconcilier les intérêts humains et la conservation dans la Réserve de Chasse du Selous ». Les données présentées sont complétées dans une seconde publication de Hahn et Kaggi (2001). On y apprend par exemple que pour la saison de chasse 1996-1997, « Vingt villages dans le district de Morogoro avec 7781 ménages et une population de 41361 habitants purent récolter plus de $13000 \mathrm{~kg}$ de viande pour une valeur de 12160 US\$ » (Ndunguru et Hahn 1998 : 5). Les auteurs nous laissent le soin de calculer qu'il s'agit in fine de 325 grammes de viande par personne et par an ou d'un équivalent de 0,30 US\$... Ailleurs, un tableau présente le revenu annuel brut moyen par village procuré par les WMA des zones tampon en 2000. Calculé sur trente-huit villages, il s'élève à 1 210,55 US\$/an (Hahn et Kaggi 2001 : 52). Il est tragique de constater que malgré leur indigence ces "bénéfices » restent significatifs au regard des budgets communaux habituels. Ainsi, le budget annuel 2008 du village d'Hassan, qui ne possède pas encore de WMA, affichait 930 euros de recettes (tableaux 9 et 10). Ici, la création d'une WMA signifierait donc plus qu'un doublement des recettes du village. Cependant, l'argument du « mieux que rien » ne saurait escamoter l'incommensurabilité qui sépare les bénéfices tirés de l'existence de la réserve, d'une part, par les différents opérateurs et, d'autre part, par les villageois.

Hahn et Kaggi nous indiquent également, incidemment, le prix auquel les villageois estiment leur propre vie : «Certains villages ont compensé financièrement les familles des scouts accidentellement tués en service par la somme de 50000 Tsh » (Hahn et Kaggi $2001: 52$ ) soit 62,50 US $\$ 33$.

L'impact ridicule de la politique participative de protection de la faune sauvage sur la vie des villageois est plus explicitement analysé par d'autres auteurs (Haller et al. 2008 ; Kidegesho 2008). Ainsi Kidegesho, pour une autre aire protégée, le Seregenti, montre que ce sont 2,5 US\$ qui reviennent annuellement à chacun des villageois (citant Emerton et Mfunda 1999). Encore faut-il remarquer, comme il le souligne, que cette somme est donnée « sous la forme de bénéfices généraux de développement comme la construction de salles de classe, de dispensaires ou de routes » (Kidegesho 2008 : 14). Cette somme ne vient pas gonfler les budgets familiaux. Mais Kidegesho pousse plus loin l'analyse de la répartition

33. Le prix d'un esclave mâle de 25 ans dans le sud des États-Unis en 1850 était de 800 US\$ (Wahl 2008). Dans l'affaire de l'attentat de Lockerbie, la Lybie versa 1,8 milliards de dollars états-uniens pour la compensation des 270 victimes, soit environ 6,7 millions par victime. La famille d'un pompier français décédé en service bénéficie d'une indemnisation correspondant à la rente d'invalidité soit une dizaine de milliers d'euros par an. 


\section{Tableau 8}

Budget du village d'Hassan (juillet 2007-juin 2008), recettes brutes $(\epsilon)$.

\begin{tabular}{|l|l|c|c|c|}
\hline \multicolumn{2}{|c|}{ Origine de la recette } & Prévisionnel & Réalisé & \% réalisé \\
\hline \multirow{2}{*}{$\begin{array}{l}\text { Taxes sur la } \\
\text { commercialisation } \\
\text { des produits de }\end{array}$} & Pêche & 345,6 & 164,22 & 47,52 \\
\cline { 2 - 5 } & Agriculture & 252 & 154,38 & 61,26 \\
\cline { 2 - 5 } & Forêt & 1150,8 & 57,36 & 4,98 \\
\hline $\begin{array}{l}\text { Petits commerces } \\
\text { sans licence }\end{array}$ & 363,6 & 9,3 & 2,56 \\
\hline Moulin & 21,6 & 0 & 0,00 \\
\hline Camping & 168 & 485,22 & 288,82 \\
\hline $\begin{array}{l}\text { Réversions } \\
\text { du Conseil du District }\end{array}$ & $\mathbf{2 5 4 1 , 6 0}$ & $\mathbf{9 2 9 , 9 4}$ & $\mathbf{3 6 , 5 9}$ \\
\hline Dons & 240 & 0 & 0 & - \\
\hline Divers & 0 & 59,46 & 0,00 \\
\hline Total & & 0 & 0 & - \\
\hline
\end{tabular}

Source : secrétariat exécutif du village d'Hassan.

Le village possède trois boutiques avec licence. C'est ici que les villageois dépensent, au jour le jour, l'essentiel de leur revenu monétaire. Les taxes versées par ces boutiques sont perçues par ... le District. Les réversions du Conseil du District concernent des subventions, des taxes et des amendes perçues par le District et dont un pourcentage devrait revenir au village. Constatant que ces versements n'ont jamais lieu, le conseil municipal ne les inclut pas dans son budget prévisionnel. 
Tableau 9

Budget du village d'Hassan (juillet 2007-juin 2008).

Dépenses (en $€$ ).

\begin{tabular}{|c|c|c|c|c|}
\hline & Nature de la dépense & Valeur & $\%$ & \\
\hline \multirow{8}{*}{ A } & Paiement des percepteurs de taxes & 297,17 & \multirow{5}{*}{63,3} & \multirow{8}{*}{91,8} \\
\hline & Indemnités & 109,92 & & \\
\hline & Nettoyage du site du camping & 19,02 & & \\
\hline & Salaires des gardiens du camping & 130,2 & & \\
\hline & Salaires des cuisiniers du camping & 32,22 & & \\
\hline & Entretien des bâtiments de la mairie & 145,74 & \multirow{3}{*}{28,5} & \\
\hline & Petites fournitures & 57,33 & & \\
\hline & Imprévus & 62,16 & & \\
\hline \multirow{3}{*}{ B } & Creusement de toilettes pour les pêcheurs & 9 & \multirow{3}{*}{\multicolumn{2}{|c|}{5}} \\
\hline & Bourse élèves secondaire & 24 & & \\
\hline & $\begin{array}{l}\text { Participation financière à la construction de l'école } \\
\text { secondaire du village voisin }\end{array}$ & 13,8 & & \\
\hline \multirow[t]{2}{*}{$\mathrm{C}$} & Épargne & 29,38 & \multicolumn{2}{|c|}{3,2} \\
\hline & Total & 929,94 & \multicolumn{2}{|c|}{100} \\
\hline
\end{tabular}

Source : secrétariat exécutif du village d'Hassan.

Nous avons regroupé les dépenses en 3 catégories A, B et C. A totalise 91,8\% des dépenses et correspond grosso modo à des frais de fonctionnement dont l'essentiel est constitué par des salaires et indemnités. $B$ et $C$ correspondent à des investissements (l'épargne est destinée à la construction d'un dispensaire) et totalisent $8,2 \%$ du budget soit 46,8 euros. 
des revenus issus de l'exploitation de la faune sauvage. «L'engagement de la Tanzanie dans la conservation est inspiré par le fait que le secteur de la faune sauvage est la base d'une industrie touristique (...) fournissant $40 \%$ des devises étrangères à la nation. Ces gains sont obtenus grâce aux concessions de chasse, à la vente des trophées, à l'exportation d'animaux vivants et au tourisme non-prédateur conduit dans les magnifiques parcs et réserves du pays » (Kidegesho 2008 : 1). L'auteur montre que les communautés rurales ne tirent quasiment aucun bénéfice des politiques de conservation de la nature, bénéfices qui sont monopolisés par les braconniers, des membres corrompus du gouvernement, les investisseurs, les ONG, les touristes étrangers, l'État, les autorités locales (comme le District et le District Council) et les riches Tanzaniens pouvant acquitter les droits de chasse ou de visite de la réserve (ibidem : 16-18)... Au contraire, ces communautés supportent l'essentiel des coûts de cette politique. Kidegesho distingue aussi les coûts d'opportunité et les coûts induits. Les premiers correspondent à la fermeture de l'accès aux ressources (telles que le gibier, les zones de culture et de pâturage, le bois d'œuvre et de chauffe, les plantes médicinales et les lieux de culte traditionnels) qu'entraîne la création d'une aire protégée (ibidem : 5). Les seconds regroupent les dommages causés par la faune sauvage : destruction des cultures, accidents corporels, prédation du bétail, transmission de maladie au bétail (ibidem : 7-13).

Ce bilan du CBNRM de la zone tampon du Selous montre que la prochaine intégration du village d'Hassan et de Saidi à une WMA n'améliorera pas a priori leur situation financière et qu'ils continueront, par nécessité, à risquer leur vie en pêchant illégalement dans la réserve. Mieux, cette nouvelle territorialisation, au sens d'Igoe et Brockington (2007), non seulement augmentera la pression déjà forte de la faune sauvage sur l'aire cultivée, mais encore, elle aura pour effet de criminaliser des pratiques de pêche aujourd'hui légales. En effet, la partie du territoire villageois devant être affectée à la WMA inclut des zones humides, temporaires ou permanentes. L'affermage prévu de la WMA à un opérateur privé est incompatible avec la pêche saisonnière traditionnelle dont elles sont l'objet.

\section{Les causes du faible impact de la politique de $\mathrm{CBC}$ sur la vie des communautés rurales du Selous}

En Tanzanie comme ailleurs, le hiatus énorme qui sépare les objectifs déclarés de la politique participative de conservation des conséquences de sa mise œuvre sur la vie des communautés rurales fait l'objet de débats.

Baldus $^{34}$ ne remet pas en cause fondamentalement le CBNRM mais considère que l'État tanzanien corrompu est le principal obstacle à sa mise en œuvre (Baldus 2009) ${ }^{35}$. S'il évoque la société civile à la suite du prix Nobel d'économie 2009 décerné à Ostrom, les solutions qu'il préconise s'apparentent aux recettes néolibérales classiques : minimiser le rôle de l'État et s'appuyer sur le secteur privé (ibidem : 42-44). Il évoque d'ailleurs fréquemment le rôle

34. Baldus est économiste, actuellement président de la Commission du Gibier Tropical du Conseil International de la Chasse et de la Conservation du Gibier (CIC). Après avoir travaillé pour le bureau de la faune sauvage à l'Union Européenne, il dirige le Selous Conservation Programme de 1987 à 1993 (financement GTZ). En 1998, à l'invitation de la Wildlife Division, il retourne en Tanzanie comme conseiller du gouvernement pour la mise en place des CBNRM et l'élaboration d'une nouvelle politique de la faune sauvage.

35. En dépit de l'utilisation fréquente et contradictoire de la rhétorique néolibérale, la lecture du rapport que Baldus a réalisé pour le compte de la FAO et du CIC est stimulante. 
régulateur du Marché. Les Community Based Organisations elles-mêmes doivent fonctionner sur le modèle entrepreneurial. Cependant, comme les compétences n'existent pas au sein de ces communautés, l'exploitation des WMA doit être confiée au secteur privé stricto sensu, « les communautés devant seulement gérer et contrôler les conditions d'affermage » (ibidem: 42). C'est aussi le secteur privé qui doit assurer la formation des villageois (ibidem : 44).

Shauri (1999) ${ }^{36}$, comme Baldus, rend l'État tanzanien responsable de l'échec du CBNRM. Il l'accuse, derrière les déclarations de principe, de perpétuer l'accaparement des ressources au détriment des communautés rurales (Shauri 1999). Malgré tout, l'État doit continuer à jouer un rôle essentiel, mais dans un cadre législatif imposant « la copropriété et la cogestion des communautés rurales et du gouvernement [et accordant une] même importance à la protection de la faune sauvage et de la biodiversité et à la défense des intérêts et des besoins des communautés rurales » (Shauri 1999 : 11). Il insiste sur la nécessité de reconnaître et de renforcer le rôle de la société civile tanzanienne (ibidem : 12).

Pour Igoe $(2007)^{37}$ les recettes néolibérales proposées par Baldus ne sauraient être une réponse aux difficultés rencontrées par les politiques de CBNRM. Au contraire, le processus de néolibéralisation, déjà en marche depuis plus de dix ans, est au cœur du problème. $\mathrm{Ce}$ processus se caractérise du point de vue juridico-politique par la re-régulation ${ }^{38}$ que l'État met en œuvre afin de créer un environnement favorable aux investisseurs privés et de consolider la position des ONG internationales de conservation de la nature. Là où l'État apporte sa souveraineté, les autres acteurs fournissent leur contribution spécifique (capitaux, caution morale et/ou scientifique). Mettre en évidence cette collaboration permet de sortir d'une critique dont seul l'État ferait les frais. Igoe précise que cette collaboration est conflictuelle notamment en raison du partage de la rente issue des aires protégées que chaque acteur tente d'accaparer. L'analyse d'Igoe gagnerait sans doute à étendre cette conflictualité à ce que Wacquant appelle, après Bourdieu et dans un tout autre contexte de néoliberalisation, les «champs bureaucratiques » (Wacquant 2010 : 152-152). En l'occurrence, la rente en question est également un enjeu pour d'autres secteurs de la bureaucratie tanzanienne (hydroélectricité, agriculture capitaliste, mines). Quoi qu'il en soit, les rapports de force font que les communautés rurales, considérées non pas comme des « citoyens ayants droits mais comme des acteurs mineurs qui ont besoin de guide et de supervision afin qu'ils ne fassent pas de mauvaises choses » (ibidem : 249), sont exclus de facto de ce partage. Les règles néolibérales (im)posées comme cadre de développement conduisent nécessairement à la rapide dépossession des communautés rurales de leur patrimoine naturel. Les villageois ne disposent en effet d'aucune des clefs qui leur ouvriraient les portes d'une gestion compétitive

36. Shauri est juriste, membre fondateur de l'ONG tanzanienne LEAT (Lawyer's Environmental Action Team) qui s'est fixé pour mission « d'assurer une saine gestion des ressources naturelles et la protection de l'environnement en Tanzanie. Elle est également impliquée dans les questions relatives à l'établissement d'un environnement politique et législatif favorisant l'émergence de la société civile, incluant les libertés civiles et les droits humains ». Shauri travaille au sein d'un cabinet d'avocats à Dar es Salaam.

37. Igoe est anthropologue à l'Université du Colorado, président de l'ONG BRIDGE (Bridge for Indigenous Development and Grassroots Empowerment) et membre des commisions « politiques environnementales, économiques et sociales » et « aires protégées » de l'UICN.

38. Igoe propose de reprendre le terme de Re-régulation introduit par Castree (2008) et non de Dé-régulation dans la mesure où « les états jouent un rôle central dans la redéfinition des ressouces naturelles de façon à les rendre accessibles aux investisseurs privés » (Igoe 2007 : 243). 
de leur capital. Leur pauvreté et la précarité de leur économie font que la privatisation du foncier ne peut conduire qu'à son aliénation (ibidem : 244). Souvent, avant même que ces mécanismes se mettent en place, l'avidité des investisseurs les prive de leurs droits formels grâce à l'autoritarisme de l'État et à la « neutralité » complice des ONG internationales et des bailleurs de fonds ${ }^{39}$.

Cependant, comme Shauri, Igoe conclut que « pour le meilleur et pour le pire, les États restent les garants ultimes des droits » (ibidem : 241) et qu'il s'agit avant tout de créer les conditions institutionnelles et réglementaires de supervision et de responsabilisation des individus et des institutions impliqués dans la conservation internationale de la nature (Ibidem : 251).

\section{Conclusion}

Le processus de néolibéralisation dont l'évolution des politiques de conservation de la nature est concomittante (Igoe et Brockington 2007) est analysé par Wacquant dans un tout autre domaine, celui des politiques sociale et pénale menées aux États-Unis et en Europe (Wacquant 2004 et 2010). Wacquant, comme Igoe, recourt au concept de re-régulation. Il qualifie de libéral-paternaliste le régime politique correspondant, "puisqu'il est libéral et permissif en haut, à l'égard des entreprises et des classes privilégiées, et paternaliste et autoritariste en bas » (Wacquant 2004 : 29), une définition qui pourrait fort bien s'appliquer à la situation tanzanienne. Considérer l'actuel processus de néolibéralisation comme un moment particulier de l'histoire du capitalisme ouvre une perspective plus large, celle, dans la longue durée, des modalités d'intégration des communautés indigènes à l'économie globale. Depuis le début de la colonisation, les populations du Rufiji ont vu se restreindre considérablement l'accès aux ressources naturelles dont l'utilisation fondait leur vie matérielle. Jusqu'aux années 1980, cette dynamique de restriction semblait sinon légitime du moins cohérente dans la mesure où les efforts continus de l'État, colonial puis postcolonial, pour soumettre les économies «traditionnelles » au marché se manifestèrent par leur assignation à une place précise, la production de cultures d'exportation (essentiellement coton puis noix de cajou). Parallèlement, les conditions d'échange exigeaient que ces économies préservent un fort secteur vivrier d'autoconsommation. L'évolution défavorable de la compétitivité des cultures d'exportation locales, accélérée par les politiques néolibérales mises en place à partir des années 1980, a conduit les populations du Rufiji à se tourner vers le marché national en accordant à la pêche une place d'abord croissante, puis quasi-exclusive, dans la nécessaire recherche d'un revenu monétaire. Il semble qu'aujourd'hui l'État n'assigne plus aucune place à ses populations dans le cadre de sa politique économique ce qui entraîne de facto son invisibilité. Cette invisibilité est flagrante dans la promotion des grands projets de développement dont le Rufiji est la cible. Bien que concurrents, ces projets manifestent tous la même absence de prise en compte de l'existence même de ces populations : un barrage hydroélectrique qui mettrait fin au régime de crue sans lequel les activités agricoles et halieutiques locales disparaissent (Hamerlynck et al. 2011) ; l'attribution de milliers

39. L'impuissance des communautés locales résulte de leur méconnaissance des lois et de l'absence de moyens financiers pour les faire valoir. Avoir des droits ne suffit pas pour les faire valoir car « in the era of free markets and free elections, scarcely anything else is free » (Igoe 2007 : 245). 
d'hectares à des investisseurs capitalistes pour la production de culture d'exportation, notamment de biofuels.

Dans ce cadre général, la proclamation d'une politique de gestion de la faune sauvage participative, qui rend les communautés rurales soudainement visibles, les désigne comme partenaires incontournables, surprend. C'est que, si les politiques de gestion de la faune sauvage sont « un type particulier de production capitaliste " (Garland 2008 : 62), il en existe bien entendu différents arrangements structuraux qui expriment des rapports de force entre différents groupes sociaux (ibidem : 62). Or, les exigences de durabilité et d'équité que les organismes internationaux ont cristallisées en paradigme de développement durable sous la pression de l'écologie politique ont également investi le champ de la conservation de la nature. La collaboration conflictuelle évoquée par Igoe exige donc la reconnaissance par l'État de la «participation » des communautés rurales. Cette reconnaissance, quoi que formelle, dresse un cadre idéologique et juridique sur lequel les communautés peuvent appuyer leurs revendications dans la mesure où, d'une part, des alliances se nouent avec d'autres secteurs de la société civile nationale et internationale, d'autre part, l'écueil de l'appropriation privée et de l'individuation des stratégies de survie est évité. Paraphrasant Shivji à propos du foncier, il faut donc s'attendre à ce que la démocratisation de la gestion de la faune sauvage soit une lutte longue et ardue. Mais les démocrates ne peuvent se permettre de l'abandonner aux technocrates et aux bureaucrates (Shivji 1998 : 109-110).

\section{Épilogue}

Si un jour vous visitez la Selous Game Reserve, vous remercierez la constance du pouvoir, colonial et postcolonial, qui a permis la construction d'une nature sauvage, purifiée de toute présence humaine et le zèle meurtrier des gardes qui la préservent. Sur la piste qui vous conduira au parc, assis(se) dans une solide voiture tout terrain climatisée ou, plus assoiffé(e) d'aventure, juché(e) sur un camion aménagé, vous traverserez sans ralentir plusieurs villages, laissant derrière vous pour seul souvenir un nuage de poussière qui n'aura pas encore fini de se déposer sur les herbes bordant la piste lorsque le ronronnement du moteur de votre véhicule deviendra inaudible aux villageois... Peut-être ferez-vous un signe amical de la main à quelques hommes jouant aux cartes, assis sur un banc de bois et entourés d'une bande de gamins. Une guirlande de sourires enfantins agitera ses mains et heureux(se) vous n'entendrez pas mon ami Mula bougonner tout en jetant avec désinvolture sa carte : ils nous prennent vraiment pour des ploucs ${ }^{40}$ !

\section{Références citées}

Ahrends, A., N.D. Burgess, S.A.H. Milledge, M.T. Bulling, B. Fisher, J.C.R. Smart, G.P Clarke., B.E. Mhoro et S.L. Lewis, 2010. « Predictable waves of sequential forest degradation and biodiversity loss spreading from an African city », Proceedings of the National Academy of Sciences 107 (33) : 14556-14561.

Azam-Ali, S.H. et E.C. Judge, 2001. Small scale cashew nut processing, FAO - http://www.fao.org/ag/ags/agsi/ Cashew/Cashew.htm (dernière consultation 20 mai 2010).

BALdus, Rolf D., 2009. « A Practical Summary of Experiences after three decades of Community-based Wildlife Conservation in Africa "What are the Lessons Learnt ?" ", Joint publication of FAO and CIC. Budapest.

40. Source : observation directe et participante à l'occasion d'une partie de carte avec Juma, Kassim et Mula le 15 juillet 2008. 
Baldus, Rolf D., Rudolf Hahn et Catherine Picard, 2009. « Community-based conservation in Tanzania », in R. Baldus (ed.), A Practical Summary of Experiences after three decades of Community-based Wildlife Conservation in Africa "What are the Lessons Learnt?", 94-100. Budapest : Joint publication of FAO and CIC.

CASTREe, Noel, 2008. «Neoliberalizing nature : the logics of deregulation and reregulation », Environment and Planning A 40 (1) : 131-152.

Duvail, Stéphanie et Olivier Hamerlynck, 2007. « The Rufiji River flood : plague or blessing ? International Journal of Biometeorology. Special issue on climate perception, 52 (1) : 33-42.

Duvail, Stéphanie, Claire Medard et Jean-Luc Paul, 2010. « Les communautés locales face aux grands projets d'aménagement des zones humides côtières en Afrique de l'Est », Politique Africaine 117 : 149-172.

EMERTon, L. et I. Mfunda, 1999. Making wildlife economically viable for communities living around the Western Serengeti, Tanzania. London : Evaluating Eden series discussion paper, 1, IIED.

FANR (Food, Agriculture and Natural Resources - SADC), 2007. Linking production and marketing chain for the development of smallholder agricultural commodities using sesame in Tanzania as a model : baseline survey report, Southern African Development Community (SADC), $17 \mathrm{p}$. http://www.sadc.int/fanr/agricresearch/icart/grantprojects/CRARF_SESAME/SesameBaselineSurvey Report_Tanzania.pdf (dernière consultation le 20 mai 2010).

GARLAND, Elizabeth, 2008. «The elephant in the room : confronting the colonial character of wildlife conservation in Africa ", African Studies Review 51 (3) : 52-74.

HAHN, Rudolph et David KagGi, 2001. « Selous game reserve development of Community Based Conservation in the buffer zone - Facts and figures ", in Rolph D. Baldus et al. (eds), Experiences with Community Based Wildlife Conservation in Tanzanie, 44-58. Dar-es-Salaam : Tanzania Wildlife Discussion Paper 29. http://www.wildlife-programme.gtz.de/wildlife/download/nr_29_3.pdf (dernière consultation le 20 mai 2010).

Haller, Tobias et al., 2008. «Who gains from community conservation? Intended and unintended costs and benefits of participative approaches in Peru and Tanzania », The Journal of Environment \& Development 17 (2) : 118-144.

HAMERLYNCK, Olivier et al., 2011. « To connect or not to connect - floods, fisheries and livelihoods in the Lower Rufiji floodplain lakes, Tanzania », Hydrological Sciences Journal.

IGOE, Jim, 2007. « Human rights, conservation and the privatization of sovereignity in Africa - a discussion of recent changes in Tanzania », Policy Matters - Conservation and Human Rights, IUCN Commission on Environmental and Social Policy, 15 juillet 2007 : 241-254.

IgOe, Jim et Dan Brockington, 2007. «Neoliberal conservation : a brief introduction », Conservation and Society, $5(4): 432-449$.

ILLIFE, John, 1979. A modern history of Tanganyika. Londres : Cambridge University Press.

Jeudy-De-Grissac, Alain et Estienne Rodary, 2009. «Protection de la nature - Aires protégées », Encyclopaedia Universalis en ligne (universalis-edu.com.kali.martinique.univ-ag.fr:5000/encyclopedie/protection-de-lanature-aires-protegees/\#) (consulté le 25/03/2011 12 : 09).

Kareiva et al., 2008. « Development en conservation goals in World Bank projects », Science 321 : 1638-1639.

Kidegesho, J.R., 2008. "Who pays for wildlife conservation in Tanzania and who benefits ? ", papier présenté à la $12^{\mathrm{e}}$ Conférence Biennale de International Association for the Study of Commons (IASC) "Governing shared resources : connecting local experience to global challenges", 14-18 juillet 2008. http://iasc2008.glos.ac.uk/conference\%20papers/papers/K/Kideghesho_102301.pdf(dernière consultation le 20 mai 2010).

KJeKshus, Helge, 1977. Ecology control and economic development in East African history ; the case of Tanganyika 1850-1950. Londres : Heinemann. 
Koponen, Juhani, 1994. Development for exploitation - German colonial policies in mainland Tanzania 1884-1914. Helsinki : Finnish Historical Society.

Latouche, Serge, 2001. « Le développement peut-il être durable », L’Ecologiste 6, Hiver 2001-2002 : 38-39.

Li, Tania M., 2002. « Engaging simplifications : community-based resource management, market processes and state agendas in upland southeast Asia », World Development 30 (2) : 265-283.

Majamba, 2001. Regulating the Hunting Industry in Tanzania - Reflections on the Legislative, Institutional and Policy-Making Frameworks. Dar-es-Salaam : Lawyer's Environmental Action Team.

http://www.leat.or.tz/publications/regulating.hunting (dernière consultation le jeudi 20 mai 2010).

Mande, Mike, 2010. « Tusks flooding out of Selous during the rainy season », The East African, march 29-april $4: 6$.

Matzke, Gordon, 1971. «African wildlife vs. people, politics and plans », Proc. Okla. Acad. Sci. 51 : 120-126.

-_-_, 1976. « The development of the Selous game reserve », Tanzania Notes and Record 79 : 37-48.

----, 1977. Wildlife in Tanzanian settlement policy : the case of the Selous. Syracuse : Syracuse University, Foreign and comparative studies/Africa series XXVIII.

MukindA, Fred et Benjamin Muindi, 2010. « Kenya used as illegal ivory trade route », Daily Nation (Kenya), Wednesday July $21: 16$.

Nelson, Fred et al., 2007. "The evolution and reform of tanzanian wildlife management », Conservation and Society 5 (2) : 232-261.

Ndunguru, Ireneus F. et Rudi Hahn, 1998. « Reconciling human interests with conservation in the Selous game reserve (Tanzania) », communication présentée au International CBNRM Workshop, Washington D.C., May 1998.

Rist, Gilbert, 2001. Le développement - Histoire d'une croyance occidentale. Paris : Presses de Science Po.

Rodgers, W.A., 1976. " Past Wangindo settlement in the Eastern Selous game reserve », Tanzania Notes and Record $77 \& 78: 21-26$.

Rufiji District, 1997. Rufiji District socio-economic. Dar-es-Salaam : Government Press.

SHAuri, Vincent, 1999. The new wildlife policy in Tanzania - Old wine in a new bottle ? Dar-es-Salaam : Lawyer's Environmental Action Team. http://www.leat.or.tz/publications/wildlife.policy (dernière consultation le jeudi 20 janvier 2010).

SHIvנ, Issa, 1998. Not yet democracy - Reforming land tenure in Tanzania. Londres : International Institute for Environment and Development.

Sundet, Geir, 1996. " The politics of land tenure in Tanzania : the modernising state vs. smallholder farmers », in Doris Schmied (ed.), Changing rural structures in Tanzania, 57-72. Münster : LIT.

WACQUANT, Loïc, 2004. Punir les pauvres - Le nouveau gouvernement de l'insécurité sociale. Marseille : Agone.

--_-, 2010. « La fabrique de l’État néolibéral », Civilisations 59 (1) : 151-174.

WAHL, Jenny, 2008. « Slavery in the United States », in Robert Whaples (ed.), EH.Net Encyclopedia. http://eh.net/encyclopedia/article/wahl.slavery.us (dernière consultation le 20 mai 2010).

Walsh, Martin, 2006. «The utilisation of wildlife in Tanzania : Key issues and experiences », papier préparé pour BEES Consulting Group (BCG), Johannesburg. 